\title{
Analytic Theory of Finite Asymptotic Expansions in the Real Domain. Part II-B: Solutions of Differential Inequalities and Asymptotic Admissibility of Standard Derivatives
}

\author{
Antonio Granata \\ Department of Mathematics and Computer Science, University of Calabria, Cosenza, Italy \\ Email: antonio.granata@unical.it
}

Received 30 April 2015; accepted 27 June 2015; published 30 June 2015

Copyright (C) 2015 by author and Scientific Research Publishing Inc.

This work is licensed under the Creative Commons Attribution International License (CC BY). http://creativecommons.org/licenses/by/4.0/

\section{(c) (i) Open Access}

\section{Abstract}

Part II-B of our work continues the factorizational theory of asymptotic expansions of type $\left(^{*}\right.$ ) $f(x)=a_{1} \phi_{1}(x)+\cdots+a_{n} \phi_{n}(x)+o\left(\phi_{n}(x)\right), x \rightarrow x_{0}, n \geq 3$ where the asymptotic scale

$\phi(x) \gg \phi_{2}(x) \gg \cdots \gg \phi_{n}(x), \quad x \rightarrow x_{0}$, is assumed to be an extended complete Chebyshev system on a one-sided neighborhood of $x_{0}$. The main result states that to each scale of this type it remains associated an important class of functions (namely that of generalized convex functions) enjoying the property that the expansion (*), if valid, is automatically formally differentiable $n-1$ times in the two special senses characterized in Part II-A. A second result shows that formal applications of ordinary derivatives to an asymptotic expansion are rarely admissible and that they may also yield skew results even for scales of powers.

\section{Keywords}

Asymptotic Expansions, Formal Differentiation of Asymptotic Expansions, Factorizations of Ordinary Differential Operators, Chebyshev Asymptotic Scales

\section{A Brief Introduction}

This is a continuation of a previous paper [1], about the factorizational theory of asymptotic expansions in the

How to cite this paper: Granata, A. (2015) Analytic Theory of Finite Asymptotic Expansions in the Real Domain. Part II-B: Solutions of Differential Inequalities and Asymptotic Admissibility of Standard Derivatives. Advances in Pure Mathematics, 5, 481-502. http://dx.doi.org/10.4236/apm.2015.58046 
real domain.

- $\$ 8$ contains the main result in the paper: to each Chebyshev asymptotic scale $\left(\phi_{1}(x), \phi_{2}(x), \cdots, \phi_{n}(x)\right)$ it remains associated an important class of functions enjoying the property that an asymptotic expansion according to this scale, if valid, is automatically formally differentiable $n-1$ times in the two special senses characterized in $\S \S 4,5$ in Part II-A. Under the regularity assumptions of the factorizational theory, this class is characterized by an $n$ th-order differential inequality whereas in the yet-to-be-developed geometric theory it will be the class of generalized convex functions as in the special case of polynomial expansions ([2], §4).

- In $\S 9$, discussing formal application of standard derivatives to an asymptotic expansion, we characterize the existence of certain polynomial expansions at an endpoint where derivatives may fail to exist and such that the growth-order estimates of the remainders of the differentiated expansions follow unexpected algebraic rules.

- $\$ 10$ contains the proofs and $\S 11$ contains a few remarks about our theory.

Whereas the results in Part II-A show that "formal differentiation of asymptotic expansions" is usually admissible only if suitable operators linked to the given scale are used, the results in this Part II-B shed further light on this classical problem by exhibiting a meaningful and not too special case wherein suitable formal differentiations are automatically admissible and by showing that standard derivatives are admissible in very special cases only and that they may yield formulas algebraically skew from a classical viewpoint.

We continue the numbering of sections and formulas in [1], but we adopt an independent numbering of the references in the bibliography. In order to agree with some classical terminology about the matter in this paper, it is convenient to specify the signs of certain Wronskians, so we list the fundamental properties of the scale we shall use taken from ([1], Def. 2.1 and Prop. 2.3):

$$
\begin{gathered}
\phi_{i} \in A C^{n-1}\left[T, x_{0}[, 1 \leq i \leq n ;\right. \\
\phi_{1}(x) \gg \phi_{2}(x) \gg \cdots \gg \phi_{n}(x), x \rightarrow x_{0}^{-} ; \\
W\left(\phi_{1}(x), \cdots, \phi_{i}(x)\right)>0 \text { on }\left[T, x_{0}[, 1 \leq i \leq n ;\right. \\
W\left(\phi_{n}(x), \phi_{n-1}(x), \cdots, \phi_{i}(x)\right) \neq 0 \text { on }\left[T, x_{0}[, 1 \leq i \leq n ;\right. \\
\phi_{i}(x) \neq 0 \text { on }\left[T, x_{0}\left[, \text { namely sign } \phi_{i}=(-1)^{i-1}, 1 \leq i \leq n .\right.\right.
\end{gathered}
$$

The operator

$$
L_{\phi_{1}, \cdots, \phi_{n}} u:=W\left(\phi_{1}(x), \cdots, \phi_{n}(x), u\right) / W\left(\phi_{1}(x), \cdots, \phi_{n}(x)\right),
$$

is the unique linear ordinary differential operator of type $(2.1)_{1,2}$, acting on the space $A C^{n-1}\left[T, x_{0}[\right.$ and such that $\operatorname{ker} L_{\phi_{1}, \cdots, \phi_{n}}=\operatorname{span}\left(\phi_{1}, \cdots, \phi_{n}\right)$. Expansions we are studying are of type

$$
f(x)=a_{1} \phi_{1}(x)+\cdots+a_{n} \phi_{n}(x)+o\left(\phi_{n}(x)\right), x \rightarrow x_{0}^{-}, n \geq 3,
$$

and we are supposing $n \geq 3$ as the two-term theory has been thoroughly studied in [3]. Operators $L_{k}$ and $M_{k}$ are defined in formulas (3.1) to (3.4) in Part II-A; properties of the $L_{k}$ 's are reported in the first few lemmas in $\S 4$ and properties of the $M_{k}$ 's are to be found in Proposition 3.1 with the signs specified by (3.19), due to our present assumption (7.3). We recall the acronym C.F. for "canonical factorization" ([1], Prop. 2.1).

\section{Absolute Convergence and Solutions of Differential Inequalities}

The theory developed in Part II-A becomes particularly simple when the involved improper integrals are absolutely convergent and still more expressive for a function $f$ satisfying the $n$ th-order differential inequality

$$
L_{\phi_{1}, \cdots, \phi_{n}}[f(x)] \geq 0 \text { a.e.on }\left[T, x_{0}[.\right.
$$

Under the assumptions (7.1) and (7.3) this is a subclass of the so-called "generalized convex functions with re- 
spect to the system $\left(\phi_{1}, \cdots, \phi_{n}\right)$ ". The nice result stated in the next theorem claims that: if such a function admits of an asymptotic expansion (7.7) then this expansion is automatically differentiable $(n-1)$ times in the senses of both relations (4.31) and (5.6).

Theorem 8.1 (Complete asymptotic expansions). If $f \in A C^{n-1}\left[T, x_{0}[\right.$ satisfies (8.1) then the following are equivalent properties:

1) There exist $(n-1)$ real numbers $a_{1}, \cdots, a_{n-1}$ such that

$$
f(x)=a_{1} \phi_{1}(x)+\cdots+a_{n-1} \phi_{n-1}(x)+O\left(\phi_{n}(x)\right), x \rightarrow x_{0}^{-} .
$$

2) There exist $n$ real numbers $a_{1}, \cdots, a_{n}$ such that

$$
f(x)=a_{1} \phi_{1}(x)+\cdots+a_{n-1} \phi_{n-1}(x)+a_{n} \phi_{n}(x)+o\left(\phi_{n}(x)\right), x \rightarrow x_{0}^{-} .
$$

3) The following set of asymptotic expansions holds true:

$$
L_{k}[f(x)]=a_{1} L_{k}\left[\phi_{1}(x)\right]+\cdots+a_{n-k} \underbrace{L_{k}\left[\phi_{n-k}(x)\right]}_{\text {constant }}+o(1), x \rightarrow x_{0}^{-}, 0 \leq k \leq n-1 ; \text { see (4.31). }
$$

4) The following set of asymptotic expansions holds true:

$$
\begin{aligned}
& M_{k}[f(x)]=a_{k+1} M_{k}\left[\phi_{k+1}(x)\right]+\ldots+a_{n} M_{k}\left[\phi_{n}(x)\right]+o\left(M_{k}\left[\phi_{n}(x)\right]\right), \\
& x \rightarrow x_{0}^{-}, 0 \leq k \leq n-1 ; \operatorname{see}(5.5)-(5.6) .
\end{aligned}
$$

5) The following integral condition is satisfied:

$$
\left.\int_{T}^{x_{0}} \frac{1}{p_{1}} \cdots \int_{t_{n-2}}^{x_{0}} \frac{1}{p_{n-1}} \int_{t_{n-1}}^{x_{0}} \frac{L_{\phi_{1}}, \cdots, \phi_{n}}{p_{n}(t)} \mathrm{d}(t)\right]<+\infty ; \text { see (4.32). }
$$

6) The following integral condition is satisfied:

$$
\int_{T}^{x_{0}} \frac{1}{q_{n}(t)} L_{\phi_{1}, \cdots, \phi_{n}}[f(t)] \mathrm{d} t<+\infty, \text { see (5.9) and (5.19). }
$$

To this list we may obviously add the other properties in Theorem 5.1 and if this is the case the remainder $R_{0}(x)$ of the expansion in (8.3) admits of both representations:

$$
\begin{aligned}
R_{0}(x) & =\frac{(-1)^{n}}{p_{0}(x)} \int_{x}^{x_{0}} \frac{1}{p_{1}} \cdots \int_{t_{n-2}}^{x_{0}} \frac{1}{p_{n-1}} \int_{t_{n-1}}^{x_{0}} \frac{L_{\phi_{1}, \cdots, \phi_{n}}[f(t)]}{p_{n}(t)} \mathrm{d} t \\
& =\frac{(-1)^{n}}{q_{0}(x)} \int_{x}^{x_{0}} \frac{1}{q_{1}} \cdots \int_{t_{n-2}}^{x_{0}} \frac{1}{q_{n-1}} \int_{t_{n-1}}^{x_{0}} \frac{L_{\phi_{1}, \cdots, \phi_{n}}[f(t)]}{q_{n}(t)} \mathrm{d} t, x \in\left[T, x_{0}[,\right.
\end{aligned}
$$

whence it follows that

$$
(-1)^{n} R_{0}(x) \geq 0 \quad \forall x \in\left[T, x_{0}[.\right.
$$

The above equivalence " 1$) \Leftrightarrow 2$ )" simply means that, under condition (8.1), a relation " $f(x)=O\left(\phi_{n}(x)\right)$, $x \rightarrow x_{0}^{-}$," implies the existence of a finite " $\lim _{x \rightarrow x_{0}^{-}} f(x) / \phi_{n}(x) \equiv a_{n}$ ".

In addition to the equivalence " 3 ) $\Leftrightarrow 4$ )" there is another remarkable circumstance wherein the two types of formal differentiations are simultaneously admissible namely when the convergence of the pertinent improper integrals is absolute.

Theorem 8.2. For $f \in A C^{n-1}\left[T, x_{0}[\right.$ the following three integral conditions are equivalent:

$$
\int_{T}^{x_{0}} \frac{1}{p_{1}} \cdots \int_{t_{n-2}}^{x_{0}} \frac{1}{p_{n-1}} \int_{t_{n-1}}^{x_{0}} \frac{\mid L_{\phi_{1}, \cdots, \phi_{n}}[f(t)]}{p_{n}(t)} \mathrm{d} t<+\infty ;
$$




$$
\begin{gathered}
\int_{T}^{x_{0}} \tilde{P}_{n}(t)\left|L_{\phi_{1}, \cdots, \phi_{n}}[f(t)]\right| \mathrm{d} t<+\infty, \\
\text { where } \tilde{P}_{n}(t):=\frac{1}{p_{n}(t)} \int_{T}^{t} \frac{\mathrm{d} t_{n-1}}{p_{n-1}\left(t_{n-1}\right)} \cdots \int_{T}^{t_{3}} \frac{\mathrm{d} t_{2}}{p_{2}\left(t_{2}\right)} \int_{T}^{t_{2}} \frac{\mathrm{d} t_{1}}{p_{1}\left(t_{1}\right)} ; \\
\int_{T}^{x_{0}} \frac{L_{\phi_{1}, \cdots, \phi_{n}}[f(t)] \mid}{q_{n}(t)} \mathrm{d} t \equiv \int_{T}^{x_{0}}\left|\frac{W\left(\phi_{1}(t), \cdots, \phi_{n-1}(t)\right)}{W\left(\phi_{1}(t), \cdots, \phi_{n}(t)\right)} L_{\phi_{1}, \cdots, \phi_{n}}[f(t)]\right| \mathrm{d} t<+\infty .
\end{gathered}
$$

Hence each of these three conditions implies both sets of asymptotic expansions (4.31) and (5.5)-(5.6) (here the signs of the Wronskians are immaterial).

An indirect brief proof of the equivalence " $(8.11) \Leftrightarrow(8.12)$ " can be based on Theorem 8.1, but it also follows from the following remarkable relation valid for any signs of the Wronskians in (7.3), (7.4):

$$
\tilde{P}_{n}(x) \sim\left|\frac{W\left(\phi_{1}(x), \cdots, \phi_{n-1}(x)\right)}{W\left(\phi_{1}(x), \cdots, \phi_{n}(x)\right)}\right| \equiv \frac{1}{q_{n}}, x \rightarrow x_{0}^{-} .
$$

Using Theorems 4.4 and 5.2, we can also get the analogues of Theorems 8.1-8.2 for incomplete asymptotic expansions and here is a concise statement, all asymptotic relations referring to $x \rightarrow x_{0}^{-}$of course.

Theorem 8.3 (Incomplete asymptotic expansions). Let $f \in A C^{n-1}\left[T, x_{0}[\right.$ satisfy (8.1) and let $i \in\{1, \cdots, n-1\}$ be fixed. Then the following are equivalent properties:

$$
\begin{gathered}
f(x)=a_{1} \phi_{1}(x)+\cdots+a_{i-1} \phi_{i-1}(x)+O\left(\phi_{i}(x)\right) ; \\
f(x)=a_{1} \phi_{1}(x)+\cdots+a_{i-1} \phi_{i-1}(x)+a_{i} \phi_{i}(x)+o\left(\phi_{i}(x)\right) ; \\
\left\{\begin{array}{l}
L_{k}[f(x)]=a_{1} L_{k}\left[\phi_{1}(x)\right]+\cdots+a_{i} L_{k}\left[\phi_{i}(x)\right]+o\left(L_{k}\left[\phi_{i}(x)\right]\right), 0 \leq k \leq n-i ; \\
L_{n-i+h}[f(x)]=a_{1} L_{n-1+h}\left[\phi_{1}(x)\right]+\cdots+a_{i-h} L_{n-i+h}\left[\phi_{i-h}(x)\right]+o(1), 0 \leq h \leq i-1,
\end{array}\right.
\end{gathered}
$$

(which last relations are written in (4.28) in an expanded form);

$$
\begin{aligned}
& \left\{\begin{array}{l}
M_{k}[f(x)]=a_{k+1} M_{k}\left[\phi_{k+1}(x)\right]+\cdots+a_{i} M_{k}\left[\phi_{i}(x)\right]+o\left(M_{k}\left[\phi_{i}(x)\right]\right), 0 \leq k \leq i-1 ; \\
M_{k}[f(x)]=O\left(\int_{T}^{x} \frac{1}{q_{k+1}} \cdots \int_{T}^{t_{n-1}} \frac{L_{\phi_{1}, \cdots, \phi_{n}}[f(t)]}{q_{n}(t)} \mathrm{d} t\right), i \leq k \leq n-2 ; \\
M_{n-1}[f(x)]=O\left(\int_{T}^{x} \frac{L_{\phi_{1}, \cdots, \phi_{n}}[f(t)]}{q_{n}(t)} \mathrm{d} t\right) ;
\end{array}\right. \\
& \int_{T}^{x_{0}} \frac{1}{p_{n-i+1}} \int_{t_{n-i+1}}^{x_{0}} \frac{1}{p_{n-i+2}} \cdots \int_{t_{n-1}}^{x_{0}} \frac{L_{\phi_{1}, \cdots, \phi_{n}}[f(t)]}{p_{n}(t)} \mathrm{d} t<+\infty ; \\
& \int_{T}^{x_{0}} \tilde{P}_{n, i}(t)\left|L_{\phi_{1}, \cdots, \phi_{n}}[f(t)]\right| \mathrm{d} t<+\infty, \\
& \text { where } \tilde{P}_{n, i}(t):=\frac{1}{p_{n}(t)} \int_{T}^{t} \frac{\mathrm{d} t_{n-1}}{p_{n-1}} \cdots \int_{T}^{t_{n-i+2}} \frac{\mathrm{d} t_{n-i+1}}{p_{n-i+1}} \text { if } i \geq 2 \text {; } \\
& \int_{T}^{x_{0}} \frac{\mathrm{d} t_{i}}{q_{i}} \int_{T}^{t_{i}} \frac{\mathrm{d} t_{i+1}}{q_{i+1}} \cdots \int_{T}^{t_{n-1}} \frac{L_{\phi_{1}, \cdots, \phi_{n}}[f(t)]}{q_{n}(t)} \mathrm{d} t<+\infty .
\end{aligned}
$$

To the foregoing list we may obviously add property 2) or property 5) in Theorem 4.4 and properties 2)-3) in 
Theorem 5.2. For $i=1$ relation (8.14) reads $f(x)=O\left(\phi_{1}(x)\right)$ and the first group of expansions in (8.17) reduces to relation in (5.26).

Notice that relation (8.13) and the definition of $\tilde{P}_{n}(x)$ in (8.11) imply that $\lim _{x \rightarrow x_{0}^{-}}\left(1 / q_{n}\right) /\left(1 / p_{n}\right)=+\infty$, hence (8.18) does not in general imply the convergence, as $x \rightarrow x_{0}$, of any of the inner integrals appearing in (8.20); it is the stronger condition (8.10) which implies the convergence af all the integrals in (8.20) (remember that, by (2.38) and (2.45), $p_{n}$ and $q_{n}$ are not subjected to any integrability constraint).

Moreover each of the " $O$ "-estimates in (8.17) is meaningful whenever the involved integral diverges as $x \rightarrow x_{0}$ i.e. whenever the asymptotic expansion in (8.15) cannot be improved by adding more meaningful terms of the form $a_{i+j} \phi_{i+j}(x)$. As soon as one of these integrals converges to a real number as $x \rightarrow x_{0}$ then we may apply the theorem with a greater value of $i$. And in the case of divergence, under the present assumption of one-signedness, it is possible to infer from condition (8.20) sharper estimates not depending on $L_{\phi_{1}, \cdots, \phi_{n}} f$.

Theorem 8.4 (Sharper estimates for $M_{k}[f(x)], i \leq k \leq n-1$ ). Under the assumptions in Theorem 8.3 let $n \geq 3,1 \leq i \leq n-2$ and suppose that all the integrals appearing in the "O"-estimates in (8.17) diverge as $x \rightarrow x_{0}$, i.e.

$$
Q_{k+1}(x):=\int_{T}^{x} \frac{\mathrm{d} t_{k+1}}{q_{k+1}} \cdots \int_{T}^{t_{n-1}} \frac{L_{\phi_{1}, \cdots, \phi_{n}}[f(t)]}{q_{n}(t)} \mathrm{d} t \rightarrow+\infty, x \rightarrow x_{0}^{-} ; i \leq k \leq n-1 .
$$

Then the estimates in (8.17) for $M_{k}[f(x)]$ can be replaced by:

$$
\begin{gathered}
M_{i}[f(x)]=o\left(\left[\int_{x}^{x_{0}} 1 / q_{i}\right]^{-1}\right), x \rightarrow x_{0}^{-} ; \\
M_{k}[f(x)]=o\left(\left[\int_{x}^{x_{0}} \frac{\mathrm{d} t_{k}}{q_{k}} \int_{t_{k}}^{x_{0}} \frac{\mathrm{d} t_{k-1}}{q_{k-1}} \cdots \int_{t_{i+1}}^{x_{0}} \frac{\mathrm{d} t_{i}}{q_{i}}\right]^{-1}\right), x \rightarrow x_{0}^{-} ; i+1 \leq k \leq n-1 .
\end{gathered}
$$

In the present context the above estimates are by no means obvious or "natural": they have been obtained by adapting the standard calculations in the proof of the Abel-Dirichlet's test for convergence of weighted improper integrals (Lemma 10.1 below). As a simple check of their validity we reobtain classical estimates for the derivatives of nth-order convex functions, and to be consistent with the meaning of $n$ in the present series of papers, namely " $n=$ dimension of the Chebyshev system $\left(\phi_{1}, \cdots, \phi_{n}\right)$ ", we state the result for convex functions of order $n-1$ according to a standard terminology.

Corollary 8.5 (Rates of increase of derivatives of higher-order convex functions). Assume that: $f \in A C^{n-1}\left[T, x_{0}\left[, \quad x_{0} \in \mathbb{R}, n \geq 2\right.\right.$ and

$$
\left\{\begin{array}{l}
f^{(n)}(x) \geq 0 \text { a.e. on }\left[T, x_{0}[\right. \\
f(x)=O\left(\left(x-x_{0}\right)^{i}\right), x \rightarrow x_{0}^{-}, \text {for some } i \in\{0,1, \cdots, n-1\} .
\end{array}\right.
$$

Then the following asymptotic relations hold true as $x \rightarrow x_{0}^{-}$:

$$
\left\{\begin{array}{l}
f(x)=a\left(x-x_{0}\right)^{i}+o\left(\left(x-x_{0}\right)^{i}\right),(\text { for a suitable constant } a), \\
f^{(k)}(x)=a D^{k}\left(x-x_{0}\right)^{i}+o\left(\left(x-x_{0}\right)^{i-k}\right), 1 \leq k \leq n-1 .
\end{array}\right.
$$

Here the asymptotic scale is: $1 \gg\left(x-x_{0}\right) \gg \cdots \gg\left(x-x_{0}\right)^{n-1}, x \rightarrow x_{0} ; M_{k} \equiv \mathrm{d}^{k} / \mathrm{d} x^{k} ;$ and $q_{i} \equiv 1$. The special case $i=0$ asserts that if an $(n-1)$ th-order convex function $f$ on $\left[T, x_{0}\left[\right.\right.$ is bounded at $x_{0}$ then as $x \rightarrow x_{0}^{-}$:

$$
f(x)=a+o(1) \text { for some constant } a ; f^{(k)}(x)=o\left(\left(x-x_{0}\right)^{-k}\right), 1 \leq k \leq n-1 .
$$

The case $f(x):=\left(x-x_{0}\right)^{i+\epsilon}$ shows that the estimates in Theorem 8.4 are the best possible, generally speaking.

The estimates in Corollary 8.5 also follow from old results by Landau, Hardy and Littlewood about differen- 
tiation of asymptotic relations involving real powers, under assumptions of monotonicity on the derivatives, results that were discussed in [4] and then extended in [5] to asymptotic expansions in real powers. The special case $i=0$ has also been obtained independently by Popoviciu ([6], p. 28). Specialization of Theorems 8.3-8.4 to the scale $x^{n} \gg \cdots \gg x \gg 1, x \rightarrow+\infty$, yields analogous estimates at $+\infty$ ([2], Th. 4.2 and Remark 1, p. 181), if use is made of a technical result ([2], Prop. 5.2, p. 183).

An important remark. In Theorem 8.1 the two types of formal differentibility $1,2, \cdots, n-1$ times are equivalent facts whereas it is not so for a generic f such that $L_{\phi_{1}, \cdots, \phi_{n}}[f(x)]$ changes sign on each deleted left neighborhood of $x_{0}$. This has been proved for polynomial expansions [2] and for real-power expansions [5] in an indirect way by expressing the two sets of differentiated expansions as suitable sets of expansions involving the standard operators $\mathrm{d}^{k} / \mathrm{d} x^{k}$; the new sets of expansions made evident that what we called "weak formal differentiability", linked to the C.F. of type (I), is indeed a weaker property than what we called "strong formal differentiability", linked to a C.F. of type (II). This will be also proved true in Part II-C, $\S 15$, for a special class of expansions including the real-power case. The same circumstance occurs for a general two-term expansion ([3]; Remarks, p. 261) but is not a self-evident fact. In each of these three cases direct proofs could be also provided working on the corresponding integral conditions. Hence in these cases the locutions of "weak or strong formal differentiation" are legitimate. But in the general theory for $n \geq 3$ we face a nontrivial situation and state

Open problem. For $n \geq 3$ consider the two types of formal differentiability characterized in Theorems 4.5 and 5.1. Investigate whether or not the property in Theorem 5.1 always implies the one in Theorem 4.5, the two properties being equivalent in the case of absolute convergence described in Theorem 8.2.

We shall not dwell on this marginal aspect of the theory though it leaves unsolved whether or not we may use representation formula (14.38), in alternative to (15.12)-(15.13), under condition (15.10).

\section{Asymptotic Admissibility of Standard Derivatives}

\subsection{Asymptotically-Admissible Operators}

Before investigating cases wherein standard derivatives $\mathrm{d}^{k} / \mathrm{d} x^{k}$ are formally applicable to an asymptotic expansion it is good to give a rigorous definition of the involved concept, cursorily treated in ([1], §3) and ([7], §3), with a few examples.

Definition 9.1 (Asymptotically-admissible operators). Let $\mathcal{L}$ be a linear operator acting between two linear spaces of real- or complex-valued functions of one real variable, $\mathcal{L}: \mathcal{S}_{1} \rightarrow \mathcal{S}_{2}$, and let $\left(\phi_{1}, \cdots, \phi_{n}\right)$ be functions in $\mathcal{S}_{1}$ forming an asymptotic scale at $x_{0}$, possibly $x_{0}^{-}$or $x_{0}^{+}$:

$$
\phi_{1}(x) \gg \phi_{2}(x) \gg \cdots \gg \phi_{n}(x), x \rightarrow x_{0},
$$

without any further regularity assumptions.

(I) (A definition valid in special cases but highlighting the concept). $\mathcal{L}$ is said to be asymptotically admissible with respect to a given asymptotic expansion

$$
f(x)=a_{1} \phi_{1}(x)+\cdots+a_{n} \phi_{n}(x)+o\left(\phi_{n}(x)\right), x \rightarrow x_{0},
$$

if its formal application to both sides of (9.2) yields a new asymptotic expansion

$$
\mathcal{L}[f(x)]=a_{1} \mathcal{L}\left[\phi_{1}(x)\right]+\cdots+a_{n} \mathcal{L}\left[\phi_{n}(x)\right]+o\left(\mathcal{L}\left[\phi_{n}(x)\right]\right), x \rightarrow x_{0},
$$

This implicitly implies that $f \in \mathcal{S}_{1}$ and that the operator $\mathcal{L}$ changes the asymptotic scale (9.1) into a new asymptotic scale

$$
\mathcal{L}\left[\phi_{1}(x)\right] \gg \mathcal{L}\left[\phi_{2}(x)\right] \gg \cdots \gg \mathcal{L}\left[\phi_{n}(x)\right], x \rightarrow x_{0} .
$$

Put in these terms the definition is well-posed if none of the functions $\mathcal{L}\left[\phi_{i}(x)\right]$ is the zero element $\mathbf{0}$ of $\mathcal{S}_{2}$ which means the function identically zero on some neighborhood $\mathcal{I}$ of $x_{0}$ if $\mathcal{S}_{2} \subset C^{0}(\mathcal{I})$. In general, to avoid inconsistencies, the definition must be modified as follows.

(II) (A general definition). First, if $\mathcal{L}\left[\phi_{i}(x)\right]=\mathbf{0} \subset \mathcal{S}_{2} \forall i$, that is if $\phi_{i} \in \operatorname{ker} \mathcal{L} \forall i$, then the concept in question is not defined. If this is not the case then we put 


$$
m:=\max \left\{i \in\{1, \cdots, n\}: \mathcal{L}\left[\phi_{i}(x)\right] \neq \mathbf{0} \subset \mathcal{S}_{2}\right\}
$$

and say that $\mathcal{L}$ is asymptotically admissible with respect to (9.2) if

$$
\begin{gathered}
\mathcal{L}\left[\phi_{1}(x)\right] \gg \mathcal{L}\left[\phi_{2}(x)\right] \gg \cdots \gg \mathcal{L}\left[\phi_{m}(x)\right], x \rightarrow x_{0}, \\
\mathcal{L}[f(x)]=a_{1} \mathcal{L}\left[\phi_{1}(x)\right]+\cdots+a_{m} \mathcal{L}\left[\phi_{m}(x)\right]+o\left(\mathcal{L}\left[\phi_{m}(x)\right]\right), x \rightarrow x_{0},
\end{gathered}
$$

after suppression of all the zero terms. An alternative locution for an asymptotically-admissible $\mathcal{L}$ is " $\mathcal{L}$ is formally applicable to the asymptotic expansion (9.2)"; and the validity of (9.6) may be expressed by saying that " $\mathcal{L}$ preserves the asymptotic hierarchy in $(9.1)$ ".

A first group of examples clarifies the necessity of specifying "after suppression of all the zero terms". In each of the following three examples the standard operator of differentiation $\mathrm{d} / \mathrm{d} x$ is asymptotically admissible according to Definition 9.1 only if all the identically-zero terms have been suppressed.

$$
\begin{gathered}
\left\{\begin{array}{l}
f_{1}(x):=x^{2}+\log x+1+x^{-1}+\mathrm{e}^{-x}, x>0, \\
\left\{\begin{array}{l}
f_{1}(x)=x^{2}+\log x+1+x^{-1}+o\left(x^{-1}\right), x \rightarrow+\infty, \\
f_{1}^{\prime}(x)=2 x+x^{-1}-x^{-2}+o\left(x^{-2}\right), x \rightarrow+\infty
\end{array}\right.
\end{array}\right. \\
\left\{\begin{array}{l}
f_{2}(x):=x^{2}+3 x+1+x^{-1}, x>0, \\
\left\{\begin{array}{l}
f_{2}(x)=x^{2}+3 x+1+o(1), x \rightarrow+\infty \\
f_{2}^{\prime}(x)=2 x+3+o(1), x \rightarrow+\infty
\end{array}\right.
\end{array}\right. \\
\left\{\begin{array}{l}
f_{3}(x):=\log x+1+\sqrt{x}+x^{2}, x>0, \\
\left\{\begin{array}{l}
f_{3}(x)=\log x+1+\sqrt{x}+o(x), x \rightarrow 0^{+}, \\
f_{3}^{\prime}(x)=x^{-1}+\frac{1}{2} x^{-1 / 2}+o(1), x \rightarrow 0^{+} .
\end{array}\right.
\end{array}\right.
\end{gathered}
$$

That the standard operator of differentiation does not preserve asymptotic hierarchies is quite elementary but a second group of examples shows that it may not preserve asymptotic hierarchies even when acting on an $n$-tuple forming a Chebyshev asymptotic scale (signs apart) on a neighborhooh of $x_{0}$ which, in the examples below, is taken as $+\infty$.

1) Elementary examples showing that if $\phi_{1} \gg \phi_{2}$ then any asymptotic contingency may occur for the pair $\left(\phi_{1}^{\prime}, \phi_{2}^{\prime}\right)$ :

$$
\begin{aligned}
& \left\{\begin{array}{l}
\phi_{1}(x):=x^{\alpha} ; \phi_{2}(x):=x^{\beta} \quad(\alpha>\beta) ; \\
\phi_{1} \gg \phi_{2}, x \rightarrow+\infty ; \phi_{1}^{\prime} \gg \phi_{2}^{\prime}, x \rightarrow+\infty .
\end{array}\right. \\
& \left\{\begin{array}{l}
\phi_{1}(x):=1+x^{-1} ; \phi_{2}(x):=x^{-1} ; \\
\phi_{1} \gg \phi_{2}, x \rightarrow+\infty ; \phi_{1}^{\prime} \sim \phi_{2}^{\prime}, x \rightarrow+\infty .
\end{array}\right. \\
& \left\{\begin{array}{l}
\phi_{1}(x):=1+x^{-2} ; \phi_{2}(x):=x^{-1} \\
\phi_{1} \gg \phi_{2}, x \rightarrow+\infty ; \phi_{2}^{\prime} \gg \phi_{1}^{\prime}, x \rightarrow+\infty .
\end{array}\right.
\end{aligned}
$$

$\left\{\begin{array}{l}\phi_{1}(x):=\frac{1}{2} x^{2}+x \sin x+\cos x ; \phi_{2}(x):=\mathrm{e}^{-x} ; \phi_{1} \gg \phi_{2}, x \rightarrow+\infty ; \\ \text { none of the two } \operatorname{limits} \lim _{x \rightarrow+\infty} \phi_{1}^{\prime}(x) / \phi_{2}^{\prime}(x), \lim _{x \rightarrow+\infty} \phi_{2}^{\prime}(x) / \phi_{1}^{\prime}(x), \\ \text { does exist in } \overline{\mathbb{R}} \text { because of : } \phi_{1}^{\prime}(x)=x(1+\cos x) .\end{array}\right.$ 
2) Examples of Chebyshev asymptotic scales $\left(\phi_{1}, \cdots, \phi_{n}\right), n \geq 3$, such that suitable permutations of $\left(\phi_{1}^{\prime}, \cdots, \phi_{n}^{\prime}\right)$ form asymptotic scales:

$$
\left\{\begin{array}{l}
\phi_{1}(x):=x+\log x ; \phi_{2}(x):=1+x^{-3} ; \phi_{3}(x):=x^{-1} ; \phi_{4}(x):=x^{-2} ; \\
\phi_{1} \gg \phi_{2} \gg \phi_{3} \gg \phi_{4}, x \rightarrow+\infty ; \phi_{1}^{\prime} \gg \phi_{3}^{\prime} \gg \phi_{4}^{\prime} \gg \phi_{2}^{\prime}, x \rightarrow+\infty .
\end{array}\right.
$$

3) Example of a Chebyshev asymptotic scale $\left(\phi_{1}, \phi_{2}, \phi_{3}\right)$ such that no permutation of $\left(\phi_{1}^{\prime}, \phi_{2}^{\prime}, \phi_{3}^{\prime}\right)$ forms an asymptotic scale:

$$
\left\{\begin{array}{l}
\phi_{1}(x):=\frac{1}{2} x^{2}+x \sin x+\cos x ; \phi_{2}(x):=\mathrm{e}^{-x} ; \phi_{3}(x):=\mathrm{e}^{-2 x} ; \\
\phi_{1} \gg \phi_{2} \gg \phi_{3}, x \rightarrow+\infty ; \sec (13.4) .
\end{array}\right.
$$

The above examples are variations on the examples in Bourbaki ([8]; Part V, §4, pp. V.22-V.23).

4) Example of a Chebyshev asymptotic scale $\left(\phi_{1}, \cdots, \phi_{n}\right)$ such that $\left(\phi_{1}^{\prime}, \cdots, \phi_{n}^{\prime}\right)$ is an asymptotic scale as well but there exists a function $f$ such that

$$
\left\{\begin{array}{l}
f(x)=\sum_{i=1}^{n} a_{i} \phi_{i}(x)+o\left(\phi_{n}(x)\right), x \rightarrow+\infty, \\
f^{\prime}(x) \text { has no asymptotic expansion of type } \sum_{i=1}^{n} a_{i} \phi_{i}^{\prime}(x) ;
\end{array}\right.
$$

and there exists a function $g$ such that

$$
\left\{\begin{array}{l}
g(x)=\sum_{i=1}^{n} b_{i} \phi_{i}(x)+o\left(\phi_{n}(x)\right), x \rightarrow+\infty, \\
g^{\prime}(x) \text { has an incomplete asymptotic expansion, say } \\
g^{\prime}(x)=\sum_{i=1}^{k} b_{i} \phi_{i}^{\prime}(x)+o\left(\phi_{k}^{\prime}(x)\right), x \rightarrow+\infty, \text { with } k<n .
\end{array}\right.
$$

Just take

$$
x^{n} \gg x^{n-1} \gg \cdots \gg x, x \rightarrow+\infty ; f(x)=g(x):=x^{2}+x+\sin \left(x^{\alpha}\right)
$$

with different values of $\alpha>0$.

\subsection{Asymptotic Admissibility of Standard Derivatives}

Let us ask the question: What are the natural scales granting the asymptotic admissibility of the standard operators $\mathrm{d}^{k} / \mathrm{d} x^{k}$ ? Some of the above examples show that these operators do not automatically turn a given Chebyshev asymptotic scale into an asymptotic scale and, so, a rash answer to our question might suggest a scale $\left(\phi, \phi^{\prime}, \cdots, \phi^{(n-1)}\right)$ or a scale $\left(\phi_{1}, \cdots, \phi_{n}\right)$ such that $\left(\phi_{1}^{(k)}, \cdots, \phi_{n}^{(k)}\right)$ is again an asymptotic scale but this is glaringly disproved even for the familiar scale at $+\infty, x^{n} \gg x^{n-1} \gg \cdots \gg x$, by a function such as $f(x):=x^{n}+\sin \left(x^{\alpha}\right)$, $\alpha \geq n$; here we have that $f$, but not $f^{\prime}$, admits of an asymptotic expansion with respect to the mentioned scale. Of course a special case occurs when one of the sets of operators either $L_{k}$ or $M_{k}, 1 \leq k \leq n-1$, coincide with $\mathrm{d}^{k} / \mathrm{d} x^{k}$; then our operator, which we denote by the special symbol $D_{\phi}^{n}$, admits of the factorization

$$
D_{\phi}^{n} u \equiv \phi\left[\ldots\left(\left(\frac{u}{\phi}\right)^{\prime}\right)^{\prime} \ldots\right]^{\prime} \equiv \phi\left(\frac{u}{\phi}\right)^{(n)}, n \geq 2,
$$

for some function $\phi>0$ and of class $A C^{n-1}$ on some interval; and the kernel of $D_{\phi}^{n}$ is spanned by

$$
\phi(x), x \phi(x), \cdots, x^{n-1} \phi(x) \text {. }
$$


For $x_{0} \in \mathbb{R}$ or $x_{0}=+\infty$ we have respectively the associated Chebyshev asymptotic scales:

$$
\begin{gathered}
\phi(x) \gg\left(x-x_{0}\right) \phi(x) \gg \cdots \gg\left(x-x_{0}\right)^{n-1} \phi(x), x \rightarrow x_{0} ; \\
x^{n-1} \phi(x) \gg \cdots \gg x \phi(x) \gg \phi(x), x \rightarrow+\infty .
\end{gathered}
$$

Here the operators $\mathrm{d}^{k} / \mathrm{d} x^{k}$ are to be applied not to the function $f$ whose expansion is given but to the ratio $f / \phi$. From formulas (2.7), (2.8) in Part II-A, we get the C.F.'s of $D_{\phi}^{n}$.

Lemma 9.1. (I) If $x_{0} \in \mathbb{R}$ then (9.20) is a C.F. of type (II) at $x_{0}$ whereas "the" C.F. of type (I) at $x_{0}$ is

$$
D_{\phi}^{n} u \equiv \frac{\phi(x)}{\left(x-x_{0}\right)^{n-1}}\left[\left(x-x_{0}\right)^{2}\left(\ldots\left(\left(x-x_{0}\right)^{2}\left(\frac{u}{\left(x-x_{0}\right)^{n-1} \phi(x)}\right)^{\prime}\right)^{\prime} \ldots\right)^{\prime}\right]^{\prime} .
$$

(II) If $x_{0}=+\infty$ then (9.20) is "the" C.F. of type (I) at $+\infty$ whereas a C.F. of type (II) at $+\infty$, associated to (9.23), is

$$
D_{\phi}^{n} u \equiv \frac{\phi(x)}{x^{n-1}}\left[x^{2}\left(\ldots\left(x^{2}\left(\frac{u}{x^{n-1} \phi(x)}\right)^{\prime}\right)^{\prime} \ldots\right)^{\prime}\right]^{\prime} .
$$

The reader must not think that we are now filling a few pages with trivialities about Taylor's formula; as a matter of fact if we apply our theory to the operator $D_{\phi}^{n}$ in the case $x_{0}=+\infty$ we obtain the results about asymptotic parabolas for the function $f / \phi$ whose theory is thoroughly studied in [2]. But for $x_{0} \in \mathbb{R}$ the first factorizational appproach characterizes a set of asymptotic expansions wherein (quite surprisingly) the estimates of the remainders in the differentiated expansions may follow algebraic rules different from those valid both in the case $x_{0}=+\infty$ and in the case of the standard Taylor's formula; and the second factorizational appproach gives Taylor's formula as a "limit" of Taylor's formulas which is a classical elementary result to be commented on in our context. In this last case one must pay attention to the fact that formal application of the standard derivative is in general permissible only a number of times related to the growth-order of the remainder in the given asymptotic expansion. See also "examples and a final comment" at the end of this section.

Theorem 9.2. Let: $x_{0} \in \mathbb{R} ; f, \phi \in A C^{n-1}\left[T, x_{0}\left[; \phi>0\right.\right.$ on $\left[T, x_{0}[\right.$. The kth-order weighted derivative associated to factorization (9.24) is

$$
L_{k} u:=\left(x-x_{0}\right)^{2} \cdot\left\{\left(\left(x-x_{0}\right)^{2} D\right)^{k}\left[\frac{u}{\left(x-x_{0}\right)^{n-1} \phi(x)}\right]\right\}, 1 \leq k \leq n-1, n \geq 2 ;
$$

whereas the one associated to factorization (9.20) is the standard derivative

$$
M_{k} u:=(u / \phi)^{(k)}, 1 \leq k \leq n-1, n \geq 2 ;
$$

Consider now a generic polynomial of order $i \leq n-1$ of type

$$
P_{i}(x):=\sum_{j=0}^{i} a_{j}\left(x-x_{0}\right)^{j} .
$$

(I) (The continuity property of Taylor's formula). The following are equivalent properties for a fixed $i \in\{1, \cdots, n-1\}$ :

1) The set of asymptotic expansions as $x \rightarrow x_{0}^{-}$:

$$
\left\{\begin{array}{l}
f(x) / \phi(x)=P_{i}(x)+o\left(\left(x-x_{0}\right)^{i}\right) \\
(f / \phi)^{(k)}(x)=P_{i}^{(k)}(x)+o\left(\left(x-x_{0}\right)^{i-k}\right), 1 \leq k \leq i .
\end{array}\right.
$$


As concerns the bound $k \leq i$ when comparing with Theorems 5.1-5.2 notice that, in the present setting, the $\phi_{i}$ 's of the general theory are given by $\phi_{i}(x)=\left(x-x_{0}\right)^{i-1}$.

2) The improper integral (involving $n-i$ iterated integrations)

$$
\int_{T}^{x_{0}} \mathrm{~d} t_{1} \int_{T}^{t_{1}} \mathrm{~d} t_{2} \cdots \int_{T}^{t_{n-i-1}}(f(t) / \phi(t))^{(n)} \mathrm{d} t \text { converges, }
$$

which for $i=n-1$ must be read as

$$
\int_{T}^{x_{0}}(f(t) / \phi(t))^{(n)} \mathrm{d} t \text { converges. }
$$

On account of the hypothesis $f / \phi \in A C^{n-1}\left[T, x_{0}[\right.$, condition (9.30) or (9.31) is equivalent to condition

$$
\exists \lim _{x \rightarrow x_{0}^{-}}(f / \phi)^{(i)}(x) \in \mathbb{R},
$$

which is equivalent to condition

$$
\exists \lim _{x \rightarrow x_{0}^{-}}(f / \phi)^{(j)}(x) \in \mathbb{R} \forall j \in\{0,1, \cdots, i\} \text { i.e. } f / \phi \in C^{i}\left[T, x_{0}\right] .
$$

Hence relations (9.29) are nothing but Taylor's formula of order $i$ of $f / \phi$ at $x_{0}$ together with the standard differentiated relations up to order i obtained as the "limit" of the Taylor's formula at the point $\xi$ as $\xi \rightarrow x_{0}^{-}$.

(II) (A polynomial expansion at an endpoint where derivatives may fail to exist). The following are equivalent properties for a fixed $i \in\{0, \cdots, n-1\}$ :

3) The set of aymptotic expansions as $x \rightarrow x_{0}^{-}$:

$$
\left\{\begin{array}{l}
f(x)=P_{i}(x) \phi(x)+o\left(\left(x-x_{0}\right)^{i} \phi(x)\right), \\
L_{k}[f(x)]=L_{k}\left[P_{i}(x) \phi(x)\right]+ \begin{cases}o\left(\left(x-x_{0}\right)^{k+i+1-n}\right), & 1 \leq k \leq n-1-i, \\
o(1) & \max (1 ; n-1-i) \leq k \leq n-1 .\end{cases}
\end{array}\right.
$$

4) The set of aymptotic expansions as $x \rightarrow x_{0}^{-}$:

$$
\left\{\begin{array}{l}
f(x) / \phi(x)=P_{i}(x)+o\left(\left(x-x_{0}\right)^{i}\right), \\
(f / \phi)^{(k)}(x)=P_{i}^{(k)}(x)+ \begin{cases}o\left(\left(x-x_{0}\right)^{i-k}\right), & 0 \leq k \leq n-1-i \\
o\left(\left(x-x_{0}\right)^{n-1-2 k}\right), & n-1-i \leq k \leq n-1,\end{cases}
\end{array}\right.
$$

where the two estimates coincide for $k=n-1-i$, namely

$$
(f / \phi)^{(n-1-i)}(x)=P_{i}^{(n-1-i)}(x)+o\left(\left(x-x_{0}\right)^{2 i+1-n}\right) .
$$

The nondifferentiated expansion is written differently in (9.34) than in (9.35) to correctly apply the operators $L_{k}$ as defined in (9.26). Notice that for $i=n-1$ the remainder in the second relation in (9.34) is o(1) for $1 \leq k \leq n-1$, and the remainder in the second relation in (9.35) is $o\left(\left(x-x_{0}\right)^{n-1-2 k}\right)$ for $0 \leq k \leq n-1$.

5) The iterated improper integral (involving $i+1$ integrations)

$$
\int_{T}^{x_{0}}\left(t_{1}-x_{0}\right)^{-2} \mathrm{~d} t_{1} \cdots \int_{t_{i-1}}^{x_{0}}\left(t_{i}-x_{0}\right)^{-2} \mathrm{~d} t_{i} \int_{t_{i}}^{x_{0}}\left(t-x_{0}\right)^{n-1}(f(t) / \phi(t))^{(n)} \mathrm{d} t \text { converges. }
$$

We must comment on the above claims. Part (I) is a classical elementary property which may be traced back to Walter and Ford ([9], Lemma II, p. 350), 1911, and a proof is reported in Aumann and Haupt ([10], Ch. 8, $\S 8.9 .2 .1$, pp. 235-236) valid under weaker regularity assumptions involving only the existence of the highestorder left derivative of the given function and its limit as $x \rightarrow x_{0}^{-}$. Put in geometric terms it asserts that: If the osculating parabola of a certain order $i$ at a generic point $\xi$ admits of a limit position as $\xi \rightarrow x_{0}^{-}$then this last is the left osculating parabola of order $i$ at $x_{0}$. This fact historically is the idea underlying the geometric theory of limit parabolas at $+\infty$, see $([2], \S 1)$ where the two main results characterize expansions involving remainder-estimates at $+\infty$ either of the form $f^{(k)}(x)=o\left(x^{i-k}\right), k \geq 0$, or $f^{(k)}(x)=o(1), k \geq 0$. From an al- 
gebraic viewpoint the first stronger form follows the same formal rule as in Taylor's formula (9.29) and in relations (9.35) for $i=0$, whereas the second weaker form has no counterpart for $x_{0} \in \mathbb{R}$ and $i \geq 1$. The equivalence "4) $\Leftrightarrow 5$ )" is no trivial fact and let us have a closer look at the set of relations in (9.35) which may seem strange and even incorrect at a first sight. For simplicity we put $x_{0}=0$. First, the powers appearing in the $o$-terms decrease with $k$ which amounts to say that we have worse estimates for higher derivatives; and this is a natural phenomenon. Second, if for two functions $f_{1}, f_{2}$ we have

$$
f_{1}(x)=o\left(x^{i}\right), x \rightarrow 0 ; f_{2}(x)=o\left(x^{i+1}\right), x \rightarrow 0 ;
$$

then the estimate for $f_{2}^{(k)}$ is sharper than that for $f_{1}^{(k)}$ fo each $k \in\{1, \cdots, n-1\}$. This is easily checked for $k=n-1-i$ whereas for $1 \leq k \leq n-2-i$ and for $n-i<k \leq n-1$ the two estimates coincide. This fact simply says that the estimates in (9.35) for different values of $i$ are consistent. What may seem unnatural, on the contrary, is the gap of two units between the exponents inside the last $o$-term in (9.35) corresponding to consecutive values of $k$.

Examples. The following simple examples involving oscillatory functions will reassure the doubtful readers (and the author himself was in the number). For $n=2$ and $i=1$ we have the equivalence

$$
\left\{f(x)=o(x), f^{\prime}(x)=o\left(x^{-1}\right), x \rightarrow 0\right\} \Leftrightarrow \int_{\rightarrow 0} s^{-2} \mathrm{~d} s \int_{\rightarrow 0}^{s} t f^{\prime \prime}(t) \mathrm{d} t \text { converges, }
$$

an example being provided by

$$
f(x):= \begin{cases}0 & \text { if } x=0, \\ x^{\alpha} \sin \left(x^{-1}\right) & \text { if } x>0,(1<\alpha<2),\end{cases}
$$

which is differentiable at $x=0$ but $f^{\prime}$ has no limit as $x \rightarrow 0^{+}$and is unbounded.

For $n=3$ and $i=1,2$ we have the following contingencies, assuming $f \in A C^{2}$ on a deleted neighborhood of $x=0$ :

$$
\begin{aligned}
& \left\{f(x)=o(x), f^{\prime}(x)=o(1), f^{\prime \prime}(x)=o\left(x^{-2}\right), x \rightarrow 0\right\} \\
& \Leftrightarrow \int_{\rightarrow 0}\left(t_{1}\right)^{-2} \mathrm{~d} t_{1} \int_{\rightarrow 0}^{t_{1}} t^{2} f^{\prime \prime \prime}(t) \mathrm{d} t \text { converges; } \\
& \left\{f(x)=o\left(x^{2}\right), f^{\prime}(x)=o(1), f^{\prime \prime}(x)=o\left(x^{-2}\right), x \rightarrow 0\right\} \\
& \Leftrightarrow \int_{\rightarrow 0}\left(t_{1}\right)^{-2} \mathrm{~d} t_{1} \int_{\rightarrow 0}^{t_{1}}\left(t_{2}\right)^{-2} \mathrm{~d} t_{2} \int_{\rightarrow 0}^{t_{2}} t^{2} f^{\prime \prime \prime}(t) \mathrm{d} t \text { converges. }
\end{aligned}
$$

It is obvious that in both cases $f$ can be extended so as to be of class $C^{1}$ on a complete neighborhood of $x=0$ and an example for both contingencies is provided by the function:

$$
f(x):=x^{\alpha} \sin \left(x^{-1}\right), 2<\alpha<3,
$$

for which

$$
f(x)=o\left(x^{2}\right) ; f^{\prime}(x)\left\{\begin{array}{l}
=o(1) \\
\neq o(x)
\end{array} ; f^{\prime \prime}(x)=o\left(x^{-2}\right) ; x \rightarrow 0^{+} .\right.
$$

A final comment. The discussion in this section shows that formal applications of ordinary derivatives to an asymptotic expansion is not admissible generally speaking, and even for the very special asymptotic scale (9.22) the first (but not the second) factorizational approach can give seemingly-unnatural results. It is in principle true that each of the two sets of expansions characterized in Part II-A, $\S \S 4,5$, can provide asymptotic information (not always meaningful and not necessarily expansions) for the ordinary derivatives; however this is easily achieved for the first-order derivative but is practically unmanageable for higher-order derivatives and yields no theoretical result. It is also true that for expansions in arbitrary real powers as $x \rightarrow+\infty$ two lemmas of an algebraic character permit to transform each set of expansions involving the pertinent operators $L_{k}$ or $M_{k}$ into a meaningful set of expansions involving the ordinary derivatives and here again the first factorizational approach 
yields uncommon results, ([5], Lemma 7.3, p. 196, and Lemma 7.4, p. 201); but as $x \rightarrow x_{0} \in \mathbb{R}$ a possible analogue of Theorem 9.2-(II) for arbitrary powers is complicated by the fact that it is necessary to separate many a case for the exponents. On the contrary, the use of weighted derivatives defined by canonical factorizations yields a coherent and applicable theory.

\section{Proofs}

Proof of Theorem 8.1. The only thing to be proved is the inference "1) $\Rightarrow 5) \wedge 6$ )", the other properties being included in Theorems 4.5 and 5.1. We use a procedure already used in ([2], p. 193) and in ([5], p. 213). From representation in (4.15) we get (using the simplified notation $L \equiv L_{\phi_{1}, \cdots, \phi_{n}}$ ):

$$
\frac{f(x)}{\phi_{1}(x)}-c_{1}+o(1)=\frac{1 / p_{0}(x)}{\phi_{1}(x)} \int_{T}^{x} \frac{1}{p_{1}} \cdots \int_{T}^{t_{n-2}} \frac{1}{p_{n-1}} \int_{T}^{t_{n-1}} \frac{L[f(t)]}{p_{n}(t)} \mathrm{d} t, x \in\left[T, x_{0}[.\right.
$$

By the assumption (8.2) the left-hand side has a finite limit as $x \rightarrow x_{0}$, and for the right-hand side we have:

$$
\begin{aligned}
& \lim _{x \rightarrow x_{0}^{-}} \frac{1 / p_{0}(x)}{\phi_{1}(x)} \int_{T}^{x} \frac{1}{p_{1}} \cdots \int_{T}^{t_{n-2}} \frac{1}{p_{n-1}} \int_{T}^{t_{n-1}} \frac{L[f(t)]}{p_{n}(t)} \mathrm{d} t \\
& \stackrel{\operatorname{by}(2.42) \text { and }(2.43)}{=} \frac{1}{b_{1}} \lim _{x \rightarrow x_{0}^{-}} \frac{\int_{T}^{x} \frac{1}{p_{1}} \cdots \int_{T}^{t_{n-2}} \frac{1}{p_{n-1}} \int_{T}^{t_{n-1}} L[f(t)] / p_{n}(t) \mathrm{d} t}{P_{n-1}(x) / P_{0}(x)} \\
& =\frac{1}{b_{1}} \lim _{x \rightarrow x_{0}^{-}} \frac{\int_{T}^{x} \frac{1}{p_{1}} \cdots \int_{T}^{t_{n-2}} \frac{1}{p_{n-1}} \int_{T}^{t_{n-1}} L[f(t)] / p_{n}(t) \mathrm{d} t}{\int_{T}^{x} \frac{1}{p_{1}} \cdots \int_{T}^{t_{n-2}} \frac{1}{p_{n-1}}} \\
& =\cdots=\frac{1}{b_{1}} \lim _{x \rightarrow x_{0}^{-}} \int_{T}^{x} L / p_{n},
\end{aligned}
$$

after applying L'Hospital's rule $(n-1)$ times (which is legitimate as all the denominators diverge to $+\infty$ ). By the positivity of the integrand this last limit exists in $\overline{\mathbb{R}}$ and coincides with the limit of the left-hand side in (10.1) hence it must be a real number and (4.15) can take the form:

$$
f(x)=a_{1} \phi_{1}(x)+c_{2} \phi_{2}(x)+\cdots+c_{n} \phi_{n}(x)-\frac{1}{p_{0}(x)} \int_{T}^{x} \frac{1}{p_{1}} \cdots \int_{T}^{t_{n-2}} \frac{1}{p_{n-1}} \int_{t_{n-1}}^{x_{0}} \frac{L[f(t)]}{p_{n}(t)} \mathrm{d} t, x \in\left[T, x_{0}[,\right.
$$

with suitable constants $c_{2}, \cdots, c_{n}$. From this we get:

$$
\frac{f(x)-a_{1} \phi_{1}(x)}{\phi_{2}(x)}-c_{2}+o(1)=-\frac{1 / p_{0}(x)}{\phi_{2}(x)} \int_{T}^{x} \frac{1}{p_{1}} \cdots \int_{T}^{t_{n-2}} \frac{1}{p_{n-1}} \int_{t_{n-1}}^{x_{0}} \frac{L[f(t)]}{p_{n}(t)} \mathrm{d} t, x \in\left[T, x_{0}[.\right.
$$

Here again the left-hand side has a finite limit as $x \rightarrow x_{0}$ whereas the limit of the right-hand side, by (4.1), equals:

$$
\begin{aligned}
& -\frac{1}{b_{2}} \lim _{x \rightarrow x_{0}^{-}} \frac{\int_{T}^{x} \frac{1}{p_{1}} \cdots \int_{T}^{t_{n-2}} \frac{1}{p_{n-1}} \int_{t_{n-1}}^{x_{0}} L[f(t)] / p_{n}(t) \mathrm{d} t}{P_{n-2}(x) / P_{0}(x)} \\
& =-\frac{1}{b_{2}} \lim _{x \rightarrow x_{0}^{-}} \frac{\int_{T}^{x} \frac{1}{p_{1}} \cdots \int_{T}^{t_{n-2}} \frac{1}{p_{n-1}} \int_{t_{n-1}}^{x_{0}} L[f(t)] / p_{n}(t) \mathrm{d} t}{\int_{T}^{x} \frac{1}{p_{1}} \cdots \int_{T}^{t_{n-3}} \frac{1}{p_{n-2}}} \\
& =\cdots=-\frac{1}{b_{2}} \lim _{x \rightarrow x_{0}^{-}} \int_{T}^{x} \frac{1}{p_{n-1}} \int_{t_{n-1}}^{x_{0}} L / p_{n},
\end{aligned}
$$


after applying L'Hospital's rule $(n-2)$ times. Hence this last limit, which exists in $\overline{\mathbb{R}}$, must be a real number and (10.3) can be rewritten as:

$$
\begin{aligned}
f(x)= & a_{1} \phi_{1}(x)+a_{2} \phi_{2}(x)+c_{3} \phi_{3}(x)+\cdots+c_{n} \phi_{n}(x) \\
& +\frac{1}{p_{0}(x)} \int_{T}^{x} \frac{1}{p_{1}} \cdots \int_{T}^{t_{n-3}} \frac{1}{p_{n-2}} \int_{t_{n-2}}^{x_{0}} \frac{1}{p_{n-1}} \int_{t_{n-1}}^{x_{0}} \frac{L[f(t)]}{p_{n}(t)} \mathrm{d} t, x \in\left[T, x_{0}[,\right.
\end{aligned}
$$

with suitable constants $c_{3}, \cdots, c_{n}$. It is now clear how this procedure works and by induction one can prove the validity of representation:

$$
\begin{aligned}
f(x)= & a_{1} \phi_{1}(x)+\cdots+a_{n-1} \phi_{n-1}(x)+c_{n} \phi_{n}(x) \\
& +\frac{(-1)^{n-1}}{p_{0}(x)} \int_{T}^{x} \frac{1}{p_{1}} \int_{x}^{x_{0}} \frac{1}{p_{2}} \cdots \int_{t_{n-3}}^{x_{0}} \frac{1}{p_{n-2}} \int_{t_{n-2}}^{x_{0}} \frac{1}{p_{n-1}} \int_{t_{n-1}}^{x_{0}} \frac{L[f(t)]}{p_{n}(t)} \mathrm{d} t, x \in\left[T, x_{0}[,\right.
\end{aligned}
$$

with a suitable constant $c_{n}$. As a last step we observe that (8.2) implies:

$$
\left[f(x)-a_{1} \phi_{1}(x)-\cdots-a_{n-1} \phi_{n-1}(x)\right] / \phi_{n}(x)=O(1), x \rightarrow x_{0}^{-},
$$

and (10.6) in turn implies:

$$
\begin{aligned}
& \frac{1 / p_{0}(x)}{\phi_{1}(x)} \int_{T}^{x} \frac{1}{p_{1}} \int_{x}^{x_{0}} \frac{1}{p_{2}} \cdots \int_{t_{n-2}}^{x_{0}} \frac{1}{p_{n-1}} \int_{t_{n-1}}^{x_{0}} \frac{L[f(t)]}{p_{n}(t)} \mathrm{d} t \\
& \quad \operatorname{by}(4.2) \\
& \equiv \int_{T}^{x} \frac{1}{p_{1}} \int_{x}^{x_{0}} \frac{1}{p_{2}} \cdots \int_{t_{n-2}}^{x_{0}} \frac{1}{p_{n-1}} \int_{t_{n-1}}^{x_{0}} \frac{L[f(t)]}{p_{n}(t)} \mathrm{d} t=O(1), x \rightarrow x_{0}^{-} .
\end{aligned}
$$

By the positivity of the integrand this last relation implies (8.6) and the first representation in (8.8) for $R_{0}(x)$. To prove (8.7) we apply the same ideas starting from representation (5.1) and dividing by $\phi_{1}$; recalling that $\phi_{1}=1 / q_{0}$ we get

$$
\frac{f(x)}{\phi_{1}(x)}-c_{1}+o(1)=\int_{T}^{x} \frac{1}{q_{1}} \cdots \int_{T}^{t_{n-2}} \frac{1}{q_{n-1}} \int_{T}^{t_{n-1}} \frac{L[f(t)]}{q_{n}(t)} \mathrm{d} t, x \in\left[T, x_{0}[\right.
$$

which implies

$$
\int_{T}^{x_{0}} \frac{1}{q_{1}} \int_{T}^{t_{1}} \frac{1}{q_{2}} \cdots \int_{T}^{t_{n-2}} \frac{1}{q_{n-1}} \int_{T}^{t_{n-1}} \frac{L[f(t)]}{q_{n}(t)} \mathrm{d} t<+\infty
$$

and (5.1) can be rewritten as

$$
f(x)=a_{1} \phi_{1}(x)+c_{2} \phi_{2}(x)+\cdots+c_{n} \phi_{n}(x)-\frac{1}{q_{0}(x)} \int_{x}^{x_{0}} \frac{1}{q_{1}} \int_{T}^{t_{1}} \frac{1}{q_{2}} \cdots \int_{T}^{t_{n-2}} \frac{1}{q_{n-1}} \int_{T}^{t_{n-1}} \frac{L[f(t)]}{q_{n}(t)} \mathrm{d} t,
$$

with suitable constants $c_{2}, \cdots, c_{n}$. From this we get

$$
\frac{f(x)-a_{1} \phi_{1}(x)}{\phi_{2}(x)}-c_{2}+o(1)=-\frac{\phi_{1}(x)}{\phi_{2}(x)} \int_{x}^{x_{0}} \frac{1}{q_{1}} \int_{T}^{t_{1}} \frac{1}{q_{2}} \cdots \int_{T}^{t_{n-1}} \frac{L[f(t)]}{q_{n}(t)} \mathrm{d} t .
$$

Evaluating the limit of the right-hand side by L'Hospital's rule and using formula in (2.31), $1 / q_{1}=\left(\phi_{2} / \phi_{1}\right)^{\prime}$, we get

$$
\lim _{x \rightarrow x_{0}^{-}} \frac{-\int_{x}^{x_{0}} \frac{1}{q_{1}} \int_{T}^{t_{1}} \frac{1}{q_{2}} \cdots \int_{T}^{t_{n-1}} L[f(t)] / q_{n}(t) \mathrm{d} t_{H}}{\phi_{2}(x) / \phi_{1}(x)}=\lim _{x \rightarrow x_{0}^{-}} \int_{T}^{x} \frac{1}{q_{2}} \cdots \int_{T}^{t_{n-1}} L[f(t)] / q_{n}(t) \mathrm{d} t,
$$


and this last limit, which exists in $\overline{\mathbb{R}}$, must be a real number. This means that

$$
\int_{T}^{x_{0}} \frac{1}{q_{1}} \int_{t_{1}}^{x_{0}} \frac{1}{q_{2}} \int_{T}^{t_{2}} \frac{1}{q_{3}} \cdots \int_{T}^{t_{n-2}} \frac{1}{q_{n-1}} \int_{T}^{t_{n-1}} \frac{L[f(t)]}{q_{n}(t)} \mathrm{d} t<+\infty,
$$

and (10.11) can be rewritten as

$$
\begin{aligned}
f(x)= & a_{1} \phi_{1}(x)+a_{2} \phi_{2}(x)+c_{3} \phi_{3}(x)+\cdots+c_{n} \phi_{n}(x) \\
& +\frac{1}{q_{0}(x)} \int_{x}^{x_{0}} \frac{1}{q_{1}} \int_{t_{1}}^{x_{0}} \frac{1}{q_{2}} \int_{T}^{t_{2}} \frac{1}{q_{3}} \cdots \int_{T}^{t_{n-2}} \frac{1}{q_{n-1}} \int_{T}^{t_{n-1}} \frac{L[f(t)]}{q_{n}(t)} \mathrm{d} t,
\end{aligned}
$$

with suitable constants $c_{3}, \cdots, c_{n}$. For the clarity's sake we make explicit the steps in this second part of our proof. Assume by induction that the following two conditions hold true:

$$
\begin{gathered}
\int_{T}^{x_{0}} \frac{1}{q_{i}} \int_{T}^{t_{i}} \frac{1}{q_{i+1}} \cdots \int_{T}^{t_{n-2}} \frac{1}{q_{n-1}} \int_{T}^{t_{n-1}} \frac{L[f(t)]}{q_{n}(t)} \mathrm{d} t<+\infty ; \\
f(x)= \\
a_{1} \phi_{1}(x)+\cdots+a_{i} \phi_{i}(x)+c_{i+1} \phi_{i+1}(x)+\cdots+c_{n} \phi_{n}(x) \\
+\frac{(-1)^{i}}{q_{0}(x)} \int_{x}^{x_{0}} \frac{1}{q_{1}} \int_{t_{1}}^{x_{0}} \frac{1}{q_{2}} \cdots \int_{t_{i-1}}^{x_{0}} \frac{1}{q_{i}} \int_{T}^{t_{i}} \frac{1}{q_{i+1}} \cdots \int_{T}^{t_{n-1}} \frac{L[f(t)]}{q_{n}(t)} \mathrm{d} t,
\end{gathered}
$$

for some $i, 1 \leq i \leq n-2$, and suitable constants $c_{i+1}, \cdots, c_{n}$. Dividing both sides of (10.16) by $\phi_{i+1}$ and taking account of (8.2) we infer that the limit of the quantity

$$
\begin{aligned}
& {\left[\frac{(-1)^{i}}{q_{0}(x)} \int_{x}^{x_{0}} \frac{1}{q_{1}} \cdots \int_{t_{i-1}}^{x_{0}} \frac{1}{q_{i}} \int_{T}^{t_{i}} \frac{1}{q_{i+1}} \cdots \int_{T}^{t_{n-1}} \frac{L[f(t)]}{q_{n}(t)} \mathrm{d} t\right] / \phi_{i+1}(x)} \\
& \stackrel{(2.34)}{=}\left[\int_{x}^{x_{0}} \frac{1}{q_{1}} \cdots \int_{t_{i-1}}^{x_{0}} \frac{1}{q_{i}} \int_{T}^{t_{i}} \frac{1}{q_{i+1}} \cdots \int_{T}^{t_{n-1}} \frac{L[f(t)]}{q_{n}(t)} \mathrm{d} t\right] / \int_{x}^{x_{0}} \frac{1}{q_{1}} \cdots \int_{t_{i-1}}^{x_{0}} \frac{1}{q_{i}}
\end{aligned}
$$

exists in $\mathbb{R}$. Applying L'Hospital's rule $i$ times to evaluate this limit we get the new limit

$$
\lim _{x \rightarrow x_{0}^{-}} \int_{T}^{x} \frac{1}{q_{i+1}} \cdots \int_{T}^{t_{n-1}} L[f(t)] / q_{n}(t) \mathrm{d} t
$$

which, by the positivity of the integrand, exists in $\overline{\mathbb{R}}$ hence it must be a real number. We infer that condition (10.15) holds true with $i$ replaced by $i+1$ and this implies representation (10.16) with $i$ replaced by $i+1$ and suitable constants $c_{i+2}, \cdots, c_{n}$. By this inductive procedure we arrive at representation:

$$
\begin{aligned}
f(x)= & a_{1} \phi_{1}(x)+\cdots+a_{n-1} \phi_{n-1}(x)+c_{n} \phi_{n}(x) \\
& +\frac{(-1)^{n-1}}{q_{0}(x)} \int_{x}^{x_{0}} \frac{1}{q_{1}} \cdots \int_{t_{n-2}}^{x_{0}} \frac{1}{q_{n-1}} \int_{T}^{t_{n-1}} \frac{L[f(t)]}{q_{n}(t)} \mathrm{d} t, x \in\left[T, x_{0}[,\right.
\end{aligned}
$$

with some constant $c_{n}$. Dividing by $\phi_{n}$ and using (2.2) we may now conclude that

$$
\left[\int_{x}^{x_{0}} \frac{1}{q_{1}} \cdots \int_{t_{n-2}}^{x_{0}} \frac{1}{q_{n-1}} \int_{T}^{t_{n-1}} \frac{L[f(t)]}{q_{n}(t)} \mathrm{d} t\right] / \int_{x}^{x_{0}} \frac{1}{q_{1}} \cdots \int_{t_{n-2}}^{x_{0}} \frac{1}{q_{n-1}}=O(1),
$$

and if we try to evaluate the limit of the ratio on the left applying L'Hospital's rule $(n-1)$ times we get the $\lim _{x \rightarrow x_{0}^{-}} \int_{T}^{x} L[f(t)] / q_{n}(t) \mathrm{d} t$, which exists in $\overline{\mathbb{R}}$ and must be a finite number. This is condition (8.7) which allows the second representation in (8.8) for the remainder in (8.3). The proof is over.

Proof of Theorem 8.2. The equivalence between (8.10) and (8.11) easily follows from Fubini's theorem by 
interchanging the order of integrations in (8.10) whereas the equivalence between (8.11) and (8.12) is by no means an obvious fact. A concise proof based on Theorem 8.1 is as follows; putting

$$
F(x):=\frac{1}{p_{0}(x)} \int_{T}^{x} \frac{1}{p_{1}} \cdots \int_{T}^{t_{n-2}} \frac{1}{p_{n-1}} \int_{T}^{t_{n-1}} \frac{1}{p_{n}(t)} \mid L_{\phi_{1}, \cdots, \phi_{n}}[f(t)] \mathrm{d} t, x \in\left[T, x_{0}[,\right.
$$

we have

$$
F \in A C^{n-1}\left[T, x_{0}\left[; L _ { \phi _ { 1 } , \cdots , \phi _ { n } } [ F ( x ) ] = | L _ { \phi _ { 1 } , \cdots , \phi _ { n } } [ f ( x ) ] | \text { a.e.on } \left[T, x_{0}[;\right.\right.\right.
$$

hence $F$ satisfies $L_{\phi_{1}, \cdots, \phi_{n}}[F(x)] \geq 0$ a.e. on $\left[T, x_{0}[\right.$ and Theorem 8.1 implies the equivalence between (8.11) and (8.12). Now we prove relation (8.13) recalling that all the involved functions and Wronskians are strictly one-signed on the interval. The symbol of asymptotic equivalence is referred to $x \rightarrow x_{0}^{-}$of course, and, whenever used, is granted by the hierarchies of the Wronskians in (2.14) and the divergence of the involved integrals. We report a classical differentiation formula used in Proposition 2.4:

$$
\left[\frac{W\left(g_{1}(x), \cdots, g_{i-1}(x), g_{i+1}(x)\right)}{W\left(g_{1}(x), \cdots, g_{i-1}(x), g_{i}(x)\right)}\right]^{\prime}=\frac{W\left(g_{1}, \cdots, g_{i-1}\right) \cdot W\left(g_{1}, \cdots, g_{i+1}\right)}{\left[W\left(g_{1}, \cdots, g_{i}\right)\right]^{2}}, i \geq 2,
$$

valid for any ordered $(i+1)$-tuple of functions $\left(g_{1}, \cdots, g_{i}, g_{i+1}\right)$ at any point where the required derivatives exist. Now for $n=2,3$ we have:

$$
\begin{aligned}
\tilde{P}_{2}(x) & =\frac{1}{p_{2}(x)} \int_{T}^{x} \frac{\mathrm{d} t}{p_{1}(t)} \equiv p_{0}(x) p_{1}(x) \int_{T}^{x} \frac{\mathrm{d} t}{p_{1}(t)} \equiv\left|\frac{1}{\phi_{2}(x) \cdot\left(\phi_{1}(x) / \phi_{2}(x)\right)^{\prime}} \int_{T}^{x}\left(\frac{\phi_{1}}{\phi_{2}}\right)^{\prime}\right| \\
& \sim\left|\frac{\phi_{1}(x) / \phi_{2}(x)}{W\left(\phi_{2}(x), \phi_{1}(x)\right) / \phi_{2}(x)}\right|=\left|\phi_{1}(x) / W\left(\phi_{1}(x), \phi_{2}(x)\right)\right| ; \\
& \left\{\begin{array}{l}
\tilde{P}_{3}(x)=\frac{1}{p_{3}(x)} \int_{T}^{x} \frac{\mathrm{d} t_{2}}{p_{2}\left(t_{2}\right)} \int_{T}^{t_{2}} \frac{\mathrm{d} t_{1}}{p_{1}\left(t_{1}\right)} ; 1 / p_{3}=\left|W\left(\phi_{3}, \phi_{2}\right) / W\left(\phi_{3}, \phi_{2}, \phi_{1}\right)\right| ; \\
\int_{T}^{x} \frac{1}{p_{2}} \int_{T}^{t_{2}} \frac{1}{p_{1}}=\left|\phi_{T}^{x} \frac{W\left(\phi_{3}\right)^{\prime}|\sim| \phi_{2}(x) / \phi_{3}(x) \mid ;}{\left[W\left(\phi_{3}, \phi_{2}\right)\right]^{2}}\left(\int_{T}^{t} \frac{1}{p_{1}}\right) \mathrm{d} t\right| \\
\sim\left|\int_{T}^{x} \frac{\phi_{2} \cdot W\left(\phi_{2}, \phi_{3}, \phi_{1}\right)}{\left[W\left(\phi_{2}, \phi_{3}\right)\right]^{2}} \mathrm{~d} t\right| \equiv\left|\int_{T}^{x}\left[\frac{W\left(\phi_{2}, \phi_{1}\right)}{W\left(\phi_{2}, \phi_{3}\right)}\right] \mathrm{d} t\right| \sim\left|\frac{W\left(\phi_{1}, \phi_{2}\right)}{W\left(\phi_{2}, \phi_{3}\right)}\right|,
\end{array}\right.
\end{aligned}
$$

where in the last but one passage we have applied formula (10.22) to the ordered triplet $\left(\phi_{2}, \phi_{3}, \phi_{1}\right)$. Hence

$$
\tilde{P}_{3}(x) \sim\left|\frac{W\left(\phi_{3}, \phi_{2}\right)}{W\left(\phi_{3}, \phi_{2}, \phi_{1}\right)} \cdot \frac{W\left(\phi_{1}, \phi_{2}\right)}{W\left(\phi_{2}, \phi_{3}\right)}\right|=\left|\frac{W\left(\phi_{1}, \phi_{2}\right)}{W\left(\phi_{1}, \phi_{2}, \phi_{3}\right)}\right| .
$$

Now, for a fixed $n \geq 3$, we use the procedure in (10.24) to prove by induction on $i$ that

$$
\int_{T}^{x} \frac{1}{p_{i}} \cdots \int_{T}^{t_{2}} \frac{1}{p_{1}} \sim\left|\frac{W\left(\phi_{n-1}, \cdots, \phi_{n-i+1}, \phi_{n-i}\right)}{W\left(\phi_{n-1}, \cdots, \phi_{n-i+1}, \phi_{n}\right)}\right|, 2 \leq i \leq n-1 .
$$

As in (10.24) we can prove that (10.26) is true for $i=2$; assuming it to be true with $i$ replaced by $i-1$ we have: 


$$
\begin{aligned}
& \int_{T}^{x} \frac{\mathrm{d} t_{i}}{p_{i}\left(t_{i}\right)} \cdot\left[\int_{T}^{t_{i}} \frac{1}{p_{i-1}} \cdots \int_{T}^{t_{2}} \frac{1}{p_{1}}\right] \mathrm{d} t_{i} \\
& \sim\left|\int_{T}^{x} \frac{W\left(\phi_{n}, \phi_{n-1}, \cdots, \phi_{n-i+2}\right) \cdot W\left(\phi_{n}, \phi_{n-1}, \cdots, \phi_{n-i}\right)}{\left[W\left(\phi_{n}, \phi_{n-1}, \cdots, \phi_{n-i+1}\right)\right]^{2}} \cdot \frac{W\left(\phi_{n-1}, \phi_{n-2}, \cdots, \phi_{n-i+1}\right)}{W\left(\phi_{n-1}, \phi_{n-2}, \cdots, \phi_{n-i+2}, \phi_{n}\right)} \mathrm{d} t\right| \\
& \equiv\left|\int_{T}^{x} \frac{W\left(\phi_{n-1}, \phi_{n-2}, \cdots, \phi_{n-i+1}\right) \cdot W\left(\phi_{n-1}, \cdots, \phi_{n-i+1}, \phi_{n}, \phi_{n-i}\right)}{\left[W\left(\phi_{n}, \phi_{n-1}, \cdots, \phi_{n-i+1}\right)\right]^{2}} \mathrm{~d} t\right| \\
& \equiv\left|\int_{T}^{x}\left[\frac{W\left(\phi_{n-1}, \phi_{n-2}, \cdots, \phi_{n-i+1}, \phi_{n-i}\right)}{W\left(\phi_{n-1}, \cdots, \phi_{n-i+1}, \phi_{n}\right)}\right]^{\prime}\right| \mathrm{d} t|\sim| \frac{W\left(\phi_{n-1}, \phi_{n-2}, \cdots, \phi_{n-i+1}, \phi_{n-i}\right)}{W\left(\phi_{n-1}, \cdots, \phi_{n-i+1}, \phi_{n}\right)} \mid
\end{aligned}
$$

that is (10.26). Using this relation for $i=n-1$ and the expression in (2.43) for $1 / p_{n}$ we, finally, get:

$$
\tilde{P}_{n}(x) \sim\left|\frac{W\left(\phi_{n}, \phi_{n-1}, \cdots, \phi_{2}\right)}{W\left(\phi_{n}, \phi_{n-1}, \cdots, \phi_{1}\right)} \cdot \frac{W\left(\phi_{n-1}, \cdots, \phi_{1}\right)}{W\left(\phi_{n}, \phi_{n-1}, \cdots, \phi_{2}\right)}\right|,
$$

that is (8.13).

Proof of Theorem 8.3. The only thing to prove is the $O$-estimates in (8.17). From representation (5.2)

$$
\begin{aligned}
M_{i}[f(x)] & =\int_{T}^{x} \frac{1}{q_{i+1}} \cdots \int_{T}^{t_{n-1}} \frac{L[f(t)]}{q_{n}(t)} \mathrm{d} t+c_{i+1} M_{i}\left[\phi_{i+1}(x)\right]+\cdots+c_{n} M_{i}\left[\phi_{n}(x)\right] \\
& =\cdots \text { by (3.13) and (3.14) } \cdots=\int_{T}^{x} \frac{1}{q_{i+1}} \cdots \int_{T}^{t_{n-1}} \frac{L[f(t)]}{q_{n}(t)} \mathrm{d} t+c+o(1)
\end{aligned}
$$

for some constant $c$, whence the estimate for $M_{i}[f(x)]$ follows. And so on for the other estimates.

Proof of Theorem 8.4 is contained in the following lemma only valid under the stated one-signedness restrictions.

Lemma 10.1 (Growth-order estimates for iterated improper integrals with nonnegative integrands). Assumptions:

$$
\begin{gathered}
h_{i}, g \in L_{l o c}^{1}\left[T, x_{0}\left[, 1 \leq i \leq m ; T \in \mathbb{R}, x_{0} \leq+\infty ;\right.\right. \\
h_{i}>0 \text { a.e. on }\left[T, x_{0}\left[; g \geq 0 \text { a.e. on } \left[T, x_{0}[;\right.\right.\right. \\
\qquad \int_{T}^{x_{0}} h_{i}(t) \mathrm{d} t<+\infty, 1 \leq i \leq m ; \\
\left\{\begin{array}{l}
H_{i}(x):=\int_{T}^{x} h_{i}\left(t_{i}\right) \mathrm{d} t_{i} \int_{T}^{t_{i}} h_{i+1}\left(t_{i+1}\right) \mathrm{d} t_{i+1} \cdots \int_{T}^{t_{m-1}} h_{m}\left(t_{m}\right) \mathrm{d} t_{m} \int_{T}^{t_{m}} g(t) \mathrm{d} t, 1 \leq i \leq m ; \\
H_{m+1}(x):=\int_{T}^{x} g ;
\end{array}\right. \\
H_{1}(x) \text { convergent and } H_{i}(x) \text { divergent as } x \rightarrow x_{0}^{-}, 2 \leq i \leq m+1 .
\end{gathered}
$$

Thesis. The following estimates hold true:

$$
\left\{\begin{array}{l}
H_{2}(x)=o\left(\left[\int_{x}^{x_{0}} h_{1}\right]^{-1}\right), x \rightarrow x_{0}^{-} \\
H_{i}(x)=o\left(\left[\int_{x}^{x_{0}} h_{i-1} \int_{t_{i-1}}^{x_{0}} h_{i-2} \cdots \int_{t_{2}}^{x_{0}} h_{1}\right]^{-1}\right), x \rightarrow x_{0}^{-}, 2 \leq i \leq m+1 .
\end{array}\right.
$$


Proof. All functions $H_{i}$ are nonnegative and nondecreasing and satisfy $H_{i}^{\prime}(x)=h_{i}(x) H_{i+1}(x), 1 \leq i \leq m$. For $\mathrm{H}_{2}$ we have the simple estimate

$$
0 \leq H_{2}(x) \cdot \int_{x}^{x_{0}} h_{1}(t) \mathrm{d} t \leq \int_{x}^{x_{0}} h_{1}(t) H_{2}(t) \mathrm{d} t=\cdots \text { by the convergence of } H_{1}(x) \cdots=o(1),
$$

which implies the first relation in (10.33). To estimate $H_{3}(x)$ we integrate by parts as follows

$$
\int_{x}^{x_{0}} h_{1}(t) H_{2}(t) \mathrm{d} t \equiv-\int_{x}^{x_{0}} H_{2}(t) \mathrm{d}\left(\int_{t}^{x_{0}} h_{1}\right)=\left[-H_{2}(t)\left(\int_{t}^{x_{0}} h_{1}\right)\right]_{x}^{x_{0}}+\int_{x}^{x_{0}}\left(\int_{t}^{x_{0}} h_{1}\right) h_{2}(t) H_{3}(t) \mathrm{d} t
$$

Now the integral on the left is convergent by hypothesis and the first integral on the right is convergent by (10.34), hence the second integral on the right converges as well and we get the equality

$$
\int_{x}^{x_{0}} h_{1}(t) H_{2}(t) \mathrm{d} t=H_{2}(x)\left(\int_{x}^{x_{0}} h_{1}\right)+\int_{x}^{x_{0}}\left(\int_{t}^{x_{0}} h_{1}\right) h_{2}(t) H_{3}(t) \mathrm{d} t,
$$

whence, again by (10.34),

$$
\int_{x}^{x_{0}} H_{3}(t) h_{2}(t)\left(\int_{t}^{x_{0}} h_{1}\right) \mathrm{d} t=o(1) .
$$

The nondecreasingness of $H_{3}$ implies

$$
0 \leq H_{3}(x) \cdot \int_{x}^{x_{0}} h_{2}(t)\left(\int_{t}^{x_{0}} h_{1}\right) \mathrm{d} t \leq \int_{x}^{x_{0}} H_{3}(t) h_{2}(t)\left(\int_{t}^{x_{0}} h_{1}\right) \mathrm{d} t=o(1),
$$

which yields the estimate for $H_{3}$. Relations (10.36)-(10.37) give the key to proceed by induction. Putting

$$
\widetilde{H}_{i}(x):=\int_{x}^{x_{0}} h_{i-1} \int_{t_{i-1}}^{x_{0}} h_{i-2} \cdots \int_{t_{2}}^{x_{0}} h_{1},
$$

we want to prove $H_{i}(x) \cdot \widetilde{H}_{i}(x)=o(1)$. For the sake of simplicity, we write out the calculations for $H_{4}$ using (10.36), which reads $H_{3}(x) \widetilde{H}_{3}(x)=o(1)$, and integrating by parts in the integral appearing in (10.36):

$$
\begin{aligned}
& \int_{x}^{x_{0}} H_{3}(t) h_{2}(t)\left(\int_{t}^{x_{0}} h_{1}\right) \mathrm{d} t \equiv-\int_{x}^{x_{0}} H_{3}(t) \mathrm{d}\left(\widetilde{H_{3}}(t)\right) \\
& =-\left[H_{3}(t) \widetilde{H_{3}}(t)\right]_{x}^{x_{0}}+\int_{x}^{x_{0}} \widetilde{H_{3}}(t) h_{3}(t) H_{4}(t) \mathrm{d} t \\
& =H_{3}(x) \widetilde{H_{3}}(x)+\int_{x}^{x_{0}} H_{4}(t) h_{3}(t) \widetilde{H_{3}}(t) \mathrm{d} t,
\end{aligned}
$$

whence

$$
\int_{x}^{x_{0}} H_{4}(t) h_{3}(t) \widetilde{H_{3}}(t) \mathrm{d} t=o(1) .
$$

And now the nondecreasingness of $H_{4}$ implies

$$
0 \leq H_{4}(x) \cdot \underbrace{\int_{x}^{x_{0}} h_{3}(t) \widetilde{H_{3}}(t) \mathrm{d} t}_{\widetilde{H_{4}}(x)} \leq \int_{x}^{x_{0}} H_{4}(t) h_{3}(t) \widetilde{H_{3}}(t) \mathrm{d} t=o(1) .
$$

Relations (10.39)-(10.40) allow iteration of the procedure to estimate $H_{5}(x)$ and so on.

Proof of Theorem 9.2. Part (I) is nothing but Theorems 5.1 and 5.2 applied to the present case wherein $f$ is replaced by $f / \phi$ and $\phi_{i}(x) \equiv x^{i-1}$. The equivalence between (9.32) and (9.33) is an elementary fact under the tacit assumption $f(x) / \phi(x):=a_{0}$ for $x=x_{0}$. In part (II) relations (9.34) are those given in Theorems 4.4-4.5 specialized to our case and the only thing to be proved is the equivalence "3) $\Leftrightarrow 4$ )" contained in the next lemma where we simplify all formulas by assuming $x_{0}=0$ and $\phi \equiv 1$.

Lemma 10.2. Let f be of class $A C^{n-1}$ on some deleted neighborhood of $x=0$ and let 


$$
L_{k} u:=x^{2}\left(x^{2}\left(\cdots\left(x^{2}\left(x^{1-n} u\right)^{\prime}\right)^{\prime} \cdots\right)^{\prime}\right)^{\prime} \text { with } k \text { derivatives, } 1 \leq k \leq n-1, n \geq 2 .
$$

For a fixed $i \in\{0,1, \cdots, n-1\}$ the following set of asymptotic relations as $x \rightarrow 0$ :

$$
f(x)=o\left(x^{i}\right) ; L_{k}[f(x)]= \begin{cases}o\left(x^{k+i+1-n}\right), & 1 \leq k \leq n-1-i, \\ o(1), & \max (1 ; n-1-i) \leq k \leq n-1,\end{cases}
$$

is equivalent to the set of asymptotic relations, as $x \rightarrow 0$, involving only standard derivatives:

$$
f(x)=o\left(x^{i}\right) ; f^{(k)}(x)= \begin{cases}o\left(x^{i-k}\right), & 1 \leq k \leq n-1-i \\ o\left(x^{n-1-2 k}\right), & n-1-i \leq k \leq n-1,\end{cases}
$$

where the two estimates coincide for $k=n-1-i$, namely

$$
f^{(n-1-i)}(x)=o\left(x^{2 i+1-n}\right) .
$$

For $i=n-1$ in (10.42) we have $L_{k}[f(x)]=o(1)$ for all $k, 1 \leq k \leq n-1$; and in (10.43) we have $f^{(k)}(x)=o\left(x^{n-1-2 k}\right)$, for all $k, 0 \leq k \leq n-1$.

Proof. It is easily proved by induction that the expanded expressions of the $L_{k}$ 's are

$$
\left\{\begin{array}{l}
L_{1} u \equiv x^{3-n} u^{\prime}+(1-n) x^{2-n} u ; L_{2} u \equiv x^{5-n} u^{\prime \prime}+c_{2,1} x^{4-n} u^{\prime}+c_{2,0} x^{3-n} u ; \cdots ; \\
L_{k} u \equiv \sum_{j=0}^{k} c_{k, j} x^{k+j+1-n} u^{(j)}, c_{k, k}=1,
\end{array}\right.
$$

with suitable constants $c_{k, j}$ whose explicit values are not needed in our calculations. Let us now prove "(10.42) $\Rightarrow(10.43)$ '. First step. From the first equality in (10.45) we get

$$
\begin{aligned}
x^{3-n} f^{\prime}(x) & =L_{1}[f(x)]+(n-1) x^{2-n} f(x) \\
& = \begin{cases}o\left(x^{2+i-n}\right)+o\left(x^{2+i-n}\right)=o\left(x^{2+i-n}\right) & \text { if } 0 \leq i \leq n-2, \\
o(1)+o(x)=o(1) & \text { if } i=n-1,\end{cases}
\end{aligned}
$$

whence

$$
f^{\prime}(x)= \begin{cases}o\left(x^{i-1}\right) & \text { if } 0 \leq i \leq n-2, \\ o\left(x^{n-3}\right) & \text { if } i=n-1 .\end{cases}
$$

Second step. From the second equality in (10.45) we infer

$$
\begin{aligned}
& x^{5-n} f^{\prime \prime}(x)=L_{2}[f(x)]-c_{2,1} x^{4-n} f^{\prime}(x)-c_{2,0} x^{3-n} f(x) \\
& = \begin{cases}o\left(x^{3+i-n}\right)+o\left(x^{4-n+i-1}\right)+o\left(x^{3-n+i}\right)=o\left(x^{3+i-n}\right), & 0 \leq i \leq n-3, \\
o(1)+o\left(x^{4-n+i-1}\right)+o\left(x^{3-n+i}\right)=o(1), & i=n-2, \\
o(1)+o\left(x^{4-n+n-3}\right)+o\left(x^{3-n+i}\right)=o(1), & i=n-1,\end{cases}
\end{aligned}
$$

having used (10.47), and from these last estimates we get

$$
f^{\prime \prime}(x)= \begin{cases}o\left(x^{i-2}\right), & 0 \leq i \leq n-3, \\ o\left(x^{n-5}\right), & n-2 \leq i \leq n-1 .\end{cases}
$$


Suppose now that the relations in (10.43) for the derivatives have been proved true for $f^{\prime}, f^{\prime \prime}, \cdots, f^{(k)}$, $k<n-1$, and let us prove the corresponding relations for $f^{(k+1)}$. From (10.45) we get

$$
L_{k+1}[f(x)]=x^{2 k+3-n} f^{(k+1)}(x)+\sum_{j=0}^{k} c_{k+1, j} x^{k+2+j-n} f^{(j)}(x),
$$

whence

$$
x^{2 k+3-n} f^{(k+1)}(x)=L_{k+1}[f(x)]-\sum_{j=0}^{k} c_{k+1, j} x^{k+2+j-n} f^{(j)}(x) .
$$

Now for $0 \leq i \leq n-1-(k+1)$ we use the first estimate in (10.42) for $L_{k+1}[f(x)]$ and the first estimates in (10.43) for the derivatives $f^{(j)}(x)$ so getting from (10.51)

$$
x^{2 k+3-n} f^{(k+1)}(x)=o\left(x^{k+1+i+1-n}\right)+\sum_{j=0}^{k} o\left(x^{k+2+j-n+i-j}\right)=o\left(x^{k+2+i-n}\right) \text { if } 0 \leq i \leq n-k-2 .
$$

For the remaining values of $i$ we must use the second estimate in (10.42) for $L_{k+1}[f(x)]$ and the suitable estimates in (10.43) for $f^{(j)}(x)$ so getting from (10.51)

$$
x^{2 k+3-n} f^{(k+1)}(x)=o(1)-\sum_{j=0}^{k} c_{k+1, j} x^{k+2+j-n} f^{(j)}(x)=\cdots
$$

Now inside the sum each $f^{(j)}$ must be replaced by one of the two estimates in (10.43), assumed to be true, and we have two possible contingencies

$$
x^{k+2+j-n} f^{(j)}(x)= \begin{cases}o\left(x^{k+2+j-n+i-j}\right)=o\left(x^{k+2+i-n}\right) & \text { if } j \leq n-1-i, \\ o\left(x^{k+2+j-n+n-1-2 j}\right)=o\left(x^{k+1-j}\right) & \text { if } n-1-i \leq j \leq k .\end{cases}
$$

In the first case the restriction $i \geq n-k-1$ implies $k+2+i-n \geq 1$, and in the second case the restriction $j \leq k$ implies $k+1-j \geq 1$ as well. In each case the whole sum in (10.53) is $o(x)$ as $x \rightarrow 0$ and (10.53) gives

$$
x^{2 k+3-n} f^{(k+1)}(x)=o(1) \text { if } n-k-1 \leq i \leq n-1 .
$$

Finally from (10.51) we get the sought-for estimates for $f^{(k+1)}$ :

$$
f^{(k+1)}(x)= \begin{cases}o\left(x^{i-(k+1)}\right) & \text { if } 0 \leq i \leq n-(k+1)-1, \\ o\left(x^{n-1-2(k+1)}\right) & \text { if } n-(k+1) \leq i \leq n-1\end{cases}
$$

The proof of "(10.42) $\Rightarrow(10.43)$ " is over. The converse implication is checked at once replacing the estimates in (10.43) into the sum (10.45) expressing $L_{k}[f(x)]$ :

$$
\sum_{j=0}^{k} x^{k+j+1-n} \cdot\left\{\begin{array}{l}
o\left(x^{i-j}\right) \\
o\left(x^{n-1-2 j}\right)
\end{array}=\left\{\begin{array}{l}
o\left(x^{k+i+1-n}\right) \\
\sum_{j=0}^{k} o\left(x^{k-j}\right)=o(1) .
\end{array}\right.\right.
$$

\section{Some Remarks on Factorizational Theory}

\subsection{On the Use of Non-Canonical Factorizations}

We show by two examples that the use of non-C.F.'s is unreliable to construct a general theory. Let us refer, e.g., to the characterizations of an asymptotic expansion for a generalized convex function. 
First example. For $f \in A C^{2}[T,+\infty)$, we know that

$$
\left\{\begin{array}{l}
f(x)=a x^{2}+b x+c+o(1), x \rightarrow+\infty \\
f^{\prime \prime \prime}(x) \geq 0 \forall x \geq T
\end{array} \Leftrightarrow \int t^{2} f^{\prime \prime \prime}(t) \mathrm{d} t<+\infty,\right.
$$

and that the inference " $\Rightarrow$ " can be easily proved when a C.F. of the operator $L u:=u^{\prime \prime \prime}$ of type (I) at $+\infty$ is used, see ([2], proof of Th.4.2). Suppose now to use the following factorization (which is no C.F. at $+\infty$ )

$$
\left.\left.u^{\prime \prime \prime} \equiv x^{-2}\left(x^{3}\left(x^{-1} u\right)^{\prime \prime}\right)^{\prime} \forall u \in A C^{2}\right] 0,+\infty\right),
$$

and the related representation

$$
f(x)=c_{1} x^{2}+c_{2} x+c_{3}+x \int_{T}^{x} \mathrm{~d} t_{1} \int_{T}^{t_{1}} t_{2}^{-3} \mathrm{~d} t_{2} \int_{T}^{t_{2}} t^{2} f^{\prime \prime \prime}(t) \mathrm{d} t .
$$

Assuming the validity of the expansion in (11.1), we try to find a necessary integral condition involving $f^{\prime \prime \prime}$. From (11.3), we get

$$
\begin{aligned}
\mathbb{R} \stackrel{\text { by (15.4) }}{\ni} \lim _{x \rightarrow+\infty} x^{-2} f(x) & =c_{1}+\lim _{x \rightarrow+\infty}\left(\int_{T}^{x} \mathrm{~d} t_{1} \int_{T}^{t_{1}} t_{2}^{-3} \mathrm{~d} t_{2} \int_{T}^{t_{2}} t^{2} f^{\prime \prime \prime}(t) \mathrm{d} t\right) / x \\
& =c_{1}+\lim _{x \rightarrow+\infty} \int_{T}^{x} s^{-3} \mathrm{~d} s \int_{T}^{s} t^{2} f^{\prime \prime \prime}(t) \mathrm{d} t,
\end{aligned}
$$

and by reasons of constant sign, we infer

$$
\int_{T}^{+\infty} s^{-3}\left[\int_{T}^{s} t^{2} f^{\prime \prime \prime}(t) \mathrm{d} t\right] \mathrm{d} s<+\infty,
$$

a much weaker condition than $\int_{T}^{+\infty} t^{2} f^{\prime \prime \prime}(t) \mathrm{d} t<+\infty$. Hence, in this example the used factorization does not allow to characterize the expansion at hand.

Second example. Let us consider the operator $L u:=u^{\prime \prime \prime}-u^{\prime \prime}$ and the following three factorizations:

$$
\begin{gathered}
L u \equiv \mathrm{e}^{x}\left(\mathrm{e}^{-x} u^{\prime \prime}\right)^{\prime}:\left\{\begin{array}{l}
\text { a global factorization on }(-\infty,+\infty) \\
\text { and "the" C.F. of type (I) at }+\infty ;
\end{array}\right. \\
L u \equiv(x-1)^{-1}\left((x-1)^{2}\left((x-1)^{-1} \mathrm{e}^{x}\left(\mathrm{e}^{-x} u\right)^{\prime}\right)^{\prime}\right)^{\prime}:\left\{\begin{array}{l}
\text { a factorization valid on }] 1,+\infty) \\
\text { and a C.F. of type (II) at }+\infty ;
\end{array}\right. \\
L u \equiv \mathrm{e}^{x}\left(x^{-1} \mathrm{e}^{-x}\left(x^{2}\left(x^{-1} u\right)^{\prime}\right)^{\prime}\right)^{\prime}:\left\{\begin{array}{l}
\text { a factorization valid on }] 0,+\infty) \\
\text { but no C.F. at }+\infty .
\end{array}\right.
\end{gathered}
$$

We have $\operatorname{ker} L=\operatorname{span}\left(\mathrm{e}^{x} ; x ; 1\right)$ and the following characterization for an $f \in A C^{2}[T,+\infty)$

$$
\left\{\begin{array}{l}
f(x)=a \mathrm{e}^{x}+b x+c+o(1), x \rightarrow+\infty \\
f^{\prime \prime \prime}(x)-f^{\prime \prime}(x) \geq 0 \forall x \geq T
\end{array} \Leftrightarrow \int^{+\infty} t\left[f^{\prime \prime \prime}(t)-f^{\prime \prime}(t)\right] \mathrm{d} t<+\infty,\right.
$$

obtained from the results in $\S 8$ based on the use of C.F.'s. However, in this case the equivalence in (11.8) can be also obtained using the non C.F. in (11.7) if one starts from the corresponding integral representation for $f$ and reapplies the same procedure used in the proof of Theorem 8.1.

\subsection{On the Use of Integral Representations Inferred from Factorizations}

We start noticing that the convergence of an iterated integral

$$
\int_{T}^{\rightarrow x_{0}} \frac{\mathrm{d} t}{p(t)} \int_{T_{1}}^{t} g(s) \mathrm{d} s
$$


where $p>0, \int^{\rightarrow x_{0}} 1 / p=+\infty,-\infty<T \leq T_{1} \leq x_{0} \leq+\infty$, in the case of conditional convergence, may depend in an unpredictable way on the choice of $T_{1}$ as in the following two elementary examples:

$$
\begin{gathered}
\int_{1}^{+\infty} t^{-\alpha} \int_{T}^{t} \cos s \mathrm{~d} s \text { converges } \Leftrightarrow(T=k \pi, k \in \mathbb{N}) \text { if } 0<\alpha<1 ; \\
\int_{1}^{+\infty} \mathrm{d} t \int_{T}^{t}\left(s^{-1} \sin s\right)^{\prime} \mathrm{d} s \text { converges } \Leftrightarrow(T=k \pi, k \in \mathbb{Z}, \text { or } T= \pm \infty) .
\end{gathered}
$$

Now an integral representation linked to a C.F. of type (I) and more general than (4.15) in Part II-A is

$$
f(x)=c_{1} \phi_{1}(x)+\cdots+c_{n} \phi_{n}(x)+\frac{1}{p_{0}(x)} \int_{T_{1}}^{x} \frac{1}{p_{1}} \cdots \int_{T_{n-1}}^{t_{n-2}} \frac{1}{p_{n-1}} \int_{T_{n}}^{t_{n-1}} \frac{1}{p_{n}(t)} L_{\phi_{1}, \cdots, \phi_{n}}[f(t)] \mathrm{d} t, x \in\left[T, x_{0}[,\right.
$$

wherein the $c_{i}$ 's are suitable constants and the fixed endpoints $T_{i}$ are such that $T \leq T_{i} \leq x_{0}$. From this representation, one infers at once that

If there exists an $n$-tuple $\left(T_{1}, \cdots, T_{n}\right), T_{i}<x_{0}$, such that the iterated integral

$$
\int_{T_{1}}^{\rightarrow x_{0}} \frac{1}{p_{1}} \int_{T_{2}}^{t_{1}} \frac{1}{p_{2}} \cdots \int_{T_{n}}^{t_{n-1}} \frac{1}{p_{n}(t)} L_{\phi_{1}, \cdots, \phi_{n}}[f(t)] \mathrm{d} t \text { converges, }
$$

then, recalling that $\phi_{n}(x)=b_{n} / p_{0}(x)$, we get

$$
f(x)=a_{1} \phi_{1}(x)+\cdots+a_{n} \phi_{n}(x)+o\left(\phi_{n}(x)\right), x \rightarrow x_{0},
$$

with an appropriate representation of the remainder.

But, by the initial remark, such an integral condition is almost useless for general results as well as for practical applications if the $T_{i}$ 's are fixed a priori and distinct from $x_{0}$; it may be well fulfilled by some very special $f$ with an ocillatory $L f$, but it cannot be satisfied by any $f$ such that $L f>0$ no matter how small its order of growth at $x_{0}$ : in fact if the improper integral $\int_{T_{n}}^{\rightarrow x_{0}} L_{\phi_{1}, \cdots, \phi_{n}}[f(t)] / p_{n}(t) \mathrm{d} t$ represents a positive number, then the iterated integral in (11.13) diverges.

Moral. Working with the C.F. of type (I) at $x_{0}$, the sole integral conditions which can be used for sufficiently general results are those appearing in Theorem 4.4 in Part II-A.

The situation is technically different when working with a C.F. of type (II). Referring to an integral representation of type

$$
f(x)=c_{1} \phi_{1}(x)+\cdots+c_{n} \phi_{n}(x)+\frac{1}{q_{0}(x)} \int_{T_{1}}^{x} \frac{1}{q_{1}} \cdots \int_{T_{n-1}}^{t_{n-2}} \frac{1}{q_{n-1}} \int_{T_{n}}^{t_{n-1}} \frac{1}{q_{n}(t)} L_{\phi_{1}, \cdots, \phi_{n}}[f(t)] \mathrm{d} t, x \in\left[T, x_{0}[,\right.
$$

more general than (5.1) in Part II-A, we see that as soon as we may choose $T_{i}=x_{0}$ for some $i$, i.e. if some of the innermost improper integrals converge, then automatically the remaining outer integrals converge as well. Moreover, a condition like

$$
\int_{T}^{x_{0}} \frac{1}{q_{1}} \cdots \int_{t_{i-1}}^{x_{0}} \frac{1}{q_{i}} \int_{T_{i}}^{t_{i}} \frac{1}{q_{i+1}} \cdots \int_{T_{n-1}}^{t_{n-1}} \frac{1}{q_{n}(t)} L_{\phi_{1}, \cdots, \phi_{n}}[f(t)] \mathrm{d} t \text { convergent }
$$

does not "whimsically" depend on $T_{i}, \cdots, T_{n}$ if they are distinct from $x_{0}$ : if one of them is allowed to coincide with $x_{0}$, this simply means that condition (11.15) may be replaced by a stronger condition yielding additional asymptotic information.

\section{References}

[1] Granata, A. (2015) Analytic Theory of Finite Asymptotic Expansions in the Real Domain. Part II-A: The Factorizational Theory for Chebyshev Asymptotic Scales. Advances in Pure Mathematics, 5, 454-480.

[2] Granata, A. (2007) Polynomial Asymptotic Expansions in the Real Domain: The Geometric, the Factorizational, and the Stabilization Approaches. Analysis Mathematica, 33, 161-198. http://dx.doi.org/10.1007/s10476-007-0301-0

[3] Granata, A. (2011) Analytic Theory of Finite Asymptotic Expansions in the Real Domain. Part I: Two-Term Expan- 
sions of Differentiable Functions. Analysis Mathematica, 37, 245-287. http://dx.doi.org/10.1007/s10476-011-0402-7

[4] Granata, A. (2010) The Problem of Differentiating an Asymptotic Expansion in Real Powers. Part I: Unsatisfactory or Partial Results by Classical Approaches. Analysis Mathematica, 36, 85-112. http://dx.doi.org/10.1007/s10476-010-0201-6

[5] Granata, A. (2010) The Problem of Differentiating an Asymptotic Expansion in Real Powers. Part II: Factorizational Theory. Analysis Mathematica, 36, 173-218. http://dx.doi.org/10.1007/s10476-010-0301-3

[6] Popoviciu T. (1944) Les Fonctions Convexes. Hermann \& $\mathrm{C}^{\text {ie }}$ Éditeurs, Paris.

[7] Granata, A. (2015) The Factorizational Theory of Finite Asymptotic Expansions in the Real Domain: A Survey of the Main Results. Advances in Pure Mathematics, 5, 1-20. http://dx.doi.org/10.4236/apm.2015.51001

[8] Bourbaki, N. (1976) Fonctions d'une Variable Réelle-Théorie élémentaire. Hermann, Paris.

[9] Walter, M. and Ford, B. (1911) Conditions Suffisantes pour qu'une Fonction Admette un Développement Asymptotique. Bulletin de la Société Mathématique de France, 39, 347-352.

[10] Aumann, G. and Haupt, O. (1974) Einführung in die reelle Analysis. I: Funktionen einer reellen Veränderlichen. Walter de Gruyter, Berlin. http://dx.doi.org/10.1515/9783110841046 